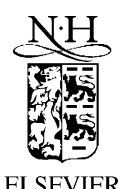

\title{
Decomposing quantum fields on branes
}

\author{
Marco Bertola ${ }^{\mathrm{a}}$, Jacques Bros ${ }^{\mathrm{c}}$, Vittorio Gorini ${ }^{\mathrm{b}}$, Ugo Moschella ${ }^{\mathrm{b}}$, \\ Richard Schaeffer ${ }^{\mathrm{c}}$ \\ ${ }^{a}$ C.R.M., Université de Montréal, C.P. 6128 Succ. Centre-Ville, Montréal (Québec), H3C 3J7 Canada \\ b Istituto di Scienze Matematiche Fisiche e Chimiche, Via Lucini 3, 22100 Como, and INFN sez. di Milano, Italy \\ c Service de Physique Théorique, C.E. Saclay, 91191 Gif-sur-Yvette, France
}

Received 17 March 2000; accepted 8 May 2000

\begin{abstract}
We provide a method to decompose the two-point function of a quantum field on a warped manifold in terms of fields living on a lower-dimensional manifold. We discuss explicit applications to Minkowski, de Sitter and anti-de Sitter quantum field theories. This decomposition presents a remarkable analogy with the holography principle, in the sense that physics in $d+1$ dimensions may be encoded into the physics in one dimension less. Moreover, in a context à la Randall-Sundrum, the method outlined here allows a mechanism of generation of mass-spectra in the 3-brane (or more generally, a $(d-1)$-brane). ( $) 2000$ Elsevier Science B.V. All rights reserved.
\end{abstract}

\section{Introduction}

The idea of dimensional reduction in quantum field theory is very old, dating back to the Kaluza-Klein theory. The motivation for considering theories in a larger ambient space is the hope to simplify or unify certain aspects of the lower-dimensional theory. Indeed, one expects that the extra degrees of freedom of the field in the ambient space survive somehow encoded in the restricted theory.

The basic ingredient of such an approach is to embed the spacetime of interest into a larger manifold and then consider an extension of the field to this ambient space in order to read off the properties of the original field into the (hopefully easier) formulation of the theory in the ambient manifold.

To make an example it is known that a QFT on the de Sitter spacetime manifests thermal properties to an inertial observer [1-4]: this is a kind of Hawking effect adapted to the present geometry. However, if we regard the de Sitter manifold as a submanifold of an ambient Minkowski (hence flat) manifold, what is an inertial observer in de Sitter becomes a uniformly accelerated observer in the ambient flat spacetime. This allows us to regard the de Sitter thermal effect as a Unruh effect in the higher-dimensional flat spacetime [5,6]. 
Moreover, the general status of field theory in flat spacetime is well established $[7,8]$, which is not true for generic curved spacetimes $[9,10]$ : the possibility of embedding the de Sitter manifold in the ambient Minkowski space allows one to formulate a sort of Wightman axiomatic framework for de Sitter spacetime, as if "geometrically" inherited from the existing axioms of the Minkowskian case [4,11]. In perspective this approach seems to be quite promising.

In the present work we address this kind of problems in the rather general framework of "warped manifolds": these are obtained by a topological product of manifolds, a "base" and a "fiber" or "leaf" (or "brane").

As a pseudo-Riemannian manifold the metric is obtained by warping the metric of the fiber by a scalar function $\omega$ depending on the point of the base.

Quite recently $[12,13]$ this sort of warped manifolds have made their appearance in the context of the "hierarchy problem". There, the study is carried out in the case in which the five dimensional background metric is made up by gluing together two slices of the five dimensional anti-de Sitter spacetime $A d S_{5}$.

The purpose of the present paper is to deal with a general situation in which the extra dimensions are warping the "brane" by an arbitrary warp factor $\omega$, which ultimately might be considered as a further degree of freedom of the full theory.

Particularly relevant is the case of only one extra dimension: under that hypothesis we will be led to the study of an auxiliary Schrödinger operator $\mathcal{L}$ in the extra dimension. Then we will prove that any (free) field $\widehat{\Phi}$ moving in the background geometry will be seen by an observer in the 3-brane as a bunch of fields $\hat{\varphi}_{\lambda}$ of different masses $m^{2}=\lambda$ : the spectrum of the allowed masses is dictated by the spectrum of the Schrödinger operator $\mathcal{L}$.

As a matter of fact the treatment does not rely in any step on the dimensionality of the embedded brane, hence we can replace the 3 -brane by any $(d-1)$-brane.

As we will see, warped products occur in quite a number of relevant examples, the first to be mentioned being the previous example of de Sitter and Minkowski. Indeed we can regard (a suitable open subset of) the flat spacetime as a warped manifold where the $d$-branes are de Sitter manifolds fibered on the half line parameterizing their curvature radius. Other examples will involve foliations of de Sitter manifolds by lower dimensional de Sitter ones, or anti-de Sitter foliated by Minkowski manifolds.

The geometric structure of these warped manifolds enables us to formulate precise correspondences between scalar Klein-Gordon fields propagating in the ambient spacetime and the restriction of them to a fixed fiber. In particular we show that under suitable assumptions and in all the examples the restricted field is a generalized free field admitting a generalized Källen-Lehmann decomposition [17] in terms of Klein-Gordon fields propagating along the $(d-1)$-brane.

The plan of the paper is the following: in Sections 1.1 and 1.2, we expose some elementary facts about the canonical Klein-Gordon theory in the flat spacetime, using it as a toy-model to introduce the ideas developed in the following.

In Section 2 we provide the general framework of Klein-Gordon QFT on warped manifolds. 
In Section 3, we enrich the previous framework by imposing appropriate "consistency conditions" between the geometries of the "bulk" and of the "brane". By using the latter, we provide in Section 4 a complete treatment of the aforementioned examples, namely of the correspondences de Sitter-Minkowski (Section 4.1), de Sitter-de Sitter (Section 4.2), Unruh-Minkowski (Section 4.3) and Minkowski-anti-de Sitter (Section 4.4). This latter application has relevance in the aforementioned context of the hierarchy problem as well as in the AdS/CFT correspondence [18] as it has been pointed out in [19].

\subsection{Canonical Klein-Gordon field theory}

We begin with a quick review of ordinary Klein-Gordon theory in Minkowskian spacetime in order to illustrate the idea of the paper.

Let us consider the $(d+1)$-dimensional Minkowski spacetime $\mathbb{M}^{d+1}$ with inertial coordinates $\left(X^{0}, X^{1}, \ldots, X^{d}\right)$ and metric

$$
\mathrm{d} s_{d+1}^{2}=\mathrm{d} X^{0^{2}}-\mathrm{d} X^{1^{2}}-\cdots-\mathrm{d} X^{d^{2}} .
$$

Let $\widehat{\Phi}$ be a Klein-Gordon quantum field of mass $M$ in the Wightman vacuum:

$$
\left(\square_{d+1}+M^{2}\right) \widehat{\Phi}=0 .
$$

The field $\widehat{\Phi}$ can be represented in terms of standard creation and annihilation operators and one deduces the momentum space (Fourier) representation for the two-points correlation function of $\widehat{\Phi}$ :

$$
\begin{aligned}
W_{M}^{(d+1)}\left(X, X^{\prime}\right) & =\left\langle\Omega, \widehat{\Phi}(X) \widehat{\Phi}\left(X^{\prime}\right) \Omega\right\rangle \\
& =\frac{1}{(2 \pi)^{d}} \int_{\mathbb{R}^{d+1}} \mathrm{e}^{-\mathrm{i} P \cdot\left(X-X^{\prime}\right)} \Theta\left(P_{0}\right) \delta\left(P^{2}-M^{2}\right) \mathrm{d}^{d+1} P,
\end{aligned}
$$

where $\Omega$ is the standard Wightman vacuum state and $\Theta$ denotes the Heaviside function. Let us consider now the restriction of the two-point function $W_{m}^{(d+1)}\left(X, X^{\prime}\right)$ to the hyperplane $\mathcal{Y}=\left\{X \in \mathbb{M}^{d+1}: X^{d}=x=\right.$ const $\} . \mathcal{Y}$ inherits its metric from the ambient Minkowski spacetime and can be identified with a $d$-dimensional Minkowski spacetime.

Since the restriction of $W_{M}^{(d+1)}\left(X, X^{\prime}\right)$ defines an acceptable two-point function (and therefore a generalized free field) on $\mathcal{Y} \simeq \mathbb{M}^{d}$, it is possible to decompose it into elementary components, namely to construct its Källen-Lehmann representation. This is particularly simple, since the representation (3) can be rewritten as follows:

$$
W_{M}^{(d+1)}\left(X, X^{\prime}\right)=\frac{1}{2 \pi} \int_{M^{2}}^{\infty} \frac{\cos \left[\sqrt{\mu^{2}-M^{2}}\left(x-x^{\prime}\right)\right]}{\sqrt{\mu^{2}-M^{2}}} W_{\mu}^{(d)}\left(y, y^{\prime}\right) \mathrm{d}\left(\mu^{2}\right),
$$

where we have introduced the notations $y=\left(y^{0}, y^{1}, \ldots, y^{d-1}\right)$ with $y^{0}=X^{0}, \ldots, y^{d-1}=$ $X^{d-1}, x=X^{d}$ and $\mu=P^{d}$. It follows that

$$
W\left(y, y^{\prime}\right)=W_{M}^{(d+1)}\left(X, X^{\prime}\right)_{\downarrow \mathcal{Y} \times \mathcal{Y}}=\frac{1}{2 \pi} \int_{\mu^{2}=M^{2}}^{\infty} W_{\mu}^{(d)}\left(y, y^{\prime}\right) \frac{\mathrm{d}\left(\mu^{2}\right)}{\sqrt{\mu^{2}-M^{2}}} .
$$


This formula is a particular instance of the well-known Källen-Lehmann decomposition.

\subsection{Spectral analysis}

Let us review this elementary example to single out its key points. First of all the Minkowski manifold $\mathbb{M}^{d+1}$ can be written as the Cartesian product $\mathbb{M}^{d+1}=\mathbb{R} \times \mathcal{Y}$. Correspondingly the metric splits into two parts $d s_{d+1}^{2}=-\mathrm{d} x^{2}+\mathrm{d} s_{d}^{2}$. This splitting allows separation of variables in the Klein-Gordon equation (2), giving rise to the following pair of equations for the modes:

$$
\begin{aligned}
& \left(\square_{d}+\lambda\right) \varphi(y)=0, \\
& \left(-\frac{\partial^{2}}{\partial x^{2}}+M^{2}\right) \theta(x)=\lambda \theta(x) .
\end{aligned}
$$

Now we can think of Eq. (7) as a spectral problem in the Hilbert space $L^{2}(\mathbb{R})$, and look for a complete set of eigenfunctions for the self-adjoint positive operator $\left(-\partial^{2} / \partial x^{2}+M^{2}\right)$, which is a Schrödinger operator with constant potential. It is useful to adopt real-valued eigenfunctions:

$$
\begin{aligned}
& \theta_{\lambda}^{1}(x)=\frac{1}{\sqrt{2 \pi \sqrt{\lambda-M^{2}}}} \cos \left(x \sqrt{\lambda-M^{2}}\right), \\
& \theta_{\lambda}^{2}(x)=\frac{1}{\sqrt{2 \pi \sqrt{\lambda-M^{2}}}} \sin \left(x \sqrt{\lambda-M^{2}}\right),
\end{aligned}
$$

with $\lambda \geqslant M^{2}$. This set of eigenfunctions is orthonormal and complete:

$$
\begin{aligned}
& \int_{\mathbb{R}} \mathrm{d} x \theta_{\lambda}^{(i)}(x) \theta_{\lambda^{\prime}}^{(j)}(x)=\delta_{i j} \delta\left(\lambda-\lambda^{\prime}\right), \\
& \sum_{i=1}^{2} \int_{M^{2}}^{\infty} \mathrm{d} \lambda \theta_{\lambda}^{(i)}(x) \theta_{\lambda}^{(i)}\left(x^{\prime}\right)=\delta\left(x-x^{\prime}\right) .
\end{aligned}
$$

Let us introduce the following "formal quantum fields":

$$
\hat{\varphi}_{\lambda}^{(i)}(y)=\int_{\mathbb{R}} \widehat{\Phi}(X) \theta_{\lambda}^{(i)}(x) \mathrm{d} x .
$$

We have used this terminology ("formal") to indicate that in the Hilbert space of the Klein-Gordon field $\widehat{\Phi}(X)$ they are operator-valued distributions not only with respect to $y$ (as usual), but also with respect to the mass parameter $\lambda$, as it will appear below explicitly in the expression of the two-point functions of these fields.

Eqs. (2) and (11) yield (in the sense of distributions in the joint variables $(y, \lambda)$ ):

$$
\left(\square_{d}+\lambda\right) \hat{\varphi}_{\lambda}^{(i)}(y)=0 .
$$

Furthermore, these fields commute with each other for different values of the parameter $\lambda$; actually, as it results from Eqs. (4) and (11), their mutual two-point correlation functions have the following expression: 


$$
W_{\lambda, \lambda^{\prime}}^{i j}\left(y, y^{\prime}\right)=\left\langle\Omega, \hat{\varphi}_{\lambda}^{(i)}(y) \hat{\varphi}_{\lambda^{\prime}}^{(j)}\left(y^{\prime}\right) \Omega\right\rangle=\delta_{i j} \delta\left(\lambda-\lambda^{\prime}\right) W_{\sqrt{\lambda}}^{(d)}\left(y, y^{\prime}\right) \Theta\left(\lambda-M^{2}\right) .
$$

By inverting Eq. (11) we obtain

$$
\widehat{\Phi}(X)=\sum_{i=1}^{2} \int_{M^{2}}^{\infty} \hat{\varphi}_{\lambda}^{(i)}(y) \theta_{\lambda}^{(i)}(x) \mathrm{d} \lambda .
$$

The previous inversion formula has been obtained by means of the completeness relation (10). It is worthwhile to stress that this is justified in the present case because the field $\widehat{\Phi}$ is a tempered operator-valued distribution and the theory of inversion of Fourier transform extends to tempered distributions [20] (namely we are making a Fourier-transform of a tempered operator-valued distribution w.r.t. the variable $x$ and taking its inversion).

A straightforward computation using Eq. (13) and Eq. (14) shows that

$$
W_{M}^{(d+1)}\left(X, X^{\prime}\right)=\sum_{i=1}^{2} \int_{M^{2}}^{\infty} \mathrm{d} \lambda \theta_{\lambda}^{(i)}(x) \theta_{\lambda}^{(i)}\left(x^{\prime}\right) W_{\sqrt{\lambda}}^{(d)}\left(y, y^{\prime}\right),
$$

formula which agrees with Eq. (4) which was worked out directly.

By restriction of the field $\widehat{\Phi}$ to the branes of constant coordinate $x=x^{\prime}$ we obtain

$$
\sum_{i=1}^{2} \int_{M^{2}}^{\infty} \mathrm{d} \lambda\left|\theta_{\lambda}^{(i)}(x)\right|^{2} W_{\sqrt{\lambda}}^{(d)}\left(y, y^{\prime}\right)=\int_{M^{2}}^{\infty} \frac{\mathrm{d} \lambda}{2 \pi \sqrt{\lambda-M^{2}}} W_{\sqrt{\lambda}}^{(d)}\left(y, y^{\prime}\right) .
$$

The spectral weight $\sum_{i=1}^{2} \leqslant\left|\theta_{\lambda}^{(i)}(x)\right|^{2}=\frac{1}{2 \pi \sqrt{\lambda-M^{2}}}$ is the density of states per unit spectrum per unit volume of the self-adjoint operator $H=-\partial^{2} / \partial x^{2}+M^{2}$.

We are going to extend this picture to more general manifolds in the following sections.

\section{Klein-Gordon fields on warped manifolds: an expansion formula}

The previous example suggests the following general structure. Let $\left(\mathcal{X},{ }^{(\mathcal{X})} g\right)$ be a Riemannian manifold, $\left(\mathcal{Y},{ }^{(\mathcal{Y})} g\right)$ a $d$-dimensional pseudo-Riemannian (Lorentzian) manifold and $\omega \in C^{\infty}\left(\mathcal{X}, \mathbb{R}^{+}\right)$be a smooth positive function. Define $\mathcal{M}=\mathcal{X} \times \mathcal{Y}$ as a topological manifold. The metric on $\mathcal{M}$ is defined by

$$
\mathrm{d} s^{2}=g_{\mu \nu} \mathrm{d} X^{\mu} \mathrm{d} X^{\nu}=\mathrm{d} s_{\mathcal{X}}^{2}+\omega^{2}(x) \mathrm{d} s_{\mathcal{Y}}^{2},
$$

where

$$
\mathrm{d} s_{\mathcal{X}}^{2}={ }^{(\mathcal{X})} g_{\mathrm{ab}} \mathrm{d} x^{\mathrm{a}} \mathrm{d} x^{\mathrm{b}}, \quad \mathrm{d} s_{\mathcal{Y}}^{2}={ }^{(\mathcal{Y})} g_{\mathrm{k} l} \mathrm{~d} y^{\mathrm{k}} \mathrm{d} y^{1} .
$$

We have denoted points of $\mathcal{Y}$ by $y$, points of $\mathcal{X}$ by $x$ and those of $\mathcal{M}$ by $X=(x, y)$ (we will use the same symbols for the corresponding coordinates); a, b are tensor indices on $\mathcal{X}$, $\mathrm{k}, 1$ on $\mathcal{Y}$ and $\mu, v$ on $\mathcal{M}$. Notice that the Riemannian metric ${ }^{(\mathcal{X})} g$ is chosen with signature $(-,-, \cdots,-)$.

The pseudo-Riemannian (Lorentzian) manifold $(\mathcal{M}, g)$ is called a warped product [21]; this structure is also denoted concisely by writing $\mathcal{M}=\mathcal{X} \times{ }_{\omega} \mathcal{Y} . \mathcal{M}$ is therefore a (trivial) 
fiber bundle over $\mathcal{X}$, whose fibers are all conformally equivalent to a manifold $\left(\mathcal{Y},{ }^{(\mathcal{Y})} g\right)$ with a conformal factor which depends only upon the basis point $x$ [22].

The simplest example of a warped product is provided by a Minkowskian background geometry (in arbitrary dimension) and it can be shown that its warped product structure can be realized in several ways, but with only two types of branes, namely either lower dimensional Minkowskian spacetimes or de Sitter spacetimes (i.e., in geometrical terms: hyperbolæ or one-sheeted hyperboloids). This can be proven by a study of the Riemann tensor (see [14-16] for the relevant formulæ in the Riemannian case, which carry over to the pseudo-Riemannian as well with obvious modifications). Similar remarks hold also for other constant curvature spacetimes.

The Laplace-Beltrami operator for 0-forms (functions) on such a manifold has the following structure:

$$
\square=\frac{1}{\sqrt{|g|}} \partial_{\mu}\left(\sqrt{|g|} g^{\mu \nu} \partial_{\nu}\right)=\Delta \mathcal{X}+\mathrm{d}\left(\partial_{\mathrm{a}} \log (\omega)\right)^{(\mathcal{X})} g^{\mathrm{ab}} \partial_{\mathrm{b}}+\frac{1}{\omega^{2}} \square \mathcal{Y} .
$$

We will assume that $\mathcal{M}$ is globally hyperbolic and consider a canonical quantum field $\widehat{\Phi}$ on $\mathcal{M}$ satisfying the Klein-Gordon equation

$$
\left(\square+M^{2}\right) \widehat{\Phi}(X)=\left(\tilde{\triangle} \mathcal{X}+\frac{1}{\omega^{2}(x)} \square \mathcal{Y}+M^{2}\right) \widehat{\Phi}(x, y)=0,
$$

where we have introduced the operator

$$
\tilde{\triangle} \mathcal{X}=\Delta \mathcal{X}+\mathrm{d}\left(\partial_{\mathrm{a}} \log \omega\right)^{(\mathcal{X})} g^{\mathrm{ab}} \partial_{\mathrm{b}}=\frac{1}{\omega^{d} \sqrt{\mid(\mathcal{X})} g \mid} \partial_{\mathrm{a}}\left(\sqrt{\left|{ }^{(\mathcal{X})} g\right|} \omega^{d(\mathcal{X})} g^{\mathrm{ab}} \partial_{\mathrm{b}}\right) .
$$

Separation of variables leads to the following equations for the modes

$$
\begin{aligned}
& (\square \mathcal{Y}+\lambda) \varphi(y)=0, \\
& \omega^{2}(x)\left(\tilde{\triangle} \mathcal{X}+M^{2}\right) \theta(x)=\lambda \theta(x) .
\end{aligned}
$$

Eq. (22) can be considered to define a spectral problem in the Hilbert space

$$
\mathcal{H}=\mathrm{L}^{2}\left(\mathcal{X}, \mathrm{d} \tilde{v}_{\mathcal{X}}\right), \quad \mathrm{d} \tilde{v}_{\mathcal{X}}(x)=\omega^{d-2}(x) \mathrm{d} v_{\mathcal{X}}(x),
$$

where $\mathrm{d} v_{\mathcal{X}}(x)=\sqrt{|\bar{X} g|} \mathrm{d} x$ is the invariant volume form on $\mathcal{X}$. Indeed, the operator $\omega^{2}(x)\left(\tilde{\triangle} \mathcal{X}+M^{2}\right)$ is symmetric on the dense domain $C_{0}^{\infty}(\mathcal{X}) \subset \mathcal{H}$. If we assume that such operator has a self-adjoint extension (which may or may not be the case in specific examples), the spectral theorem provides us with a basis $\left\{\theta_{\lambda}^{(i)}\right\}$ of generalized eigenvectors which gives a decomposition of the identity. In the same fashion as in the introductory example we then have

$$
\begin{aligned}
& \left(\theta_{\lambda}^{(i)}, \theta_{\lambda^{\prime}}^{(j)}\right)=\int_{\mathcal{X}} \bar{\theta}_{\lambda}^{(i)}(x) \theta_{\lambda^{\prime}}^{(j)}(x) \mathrm{d} \tilde{v} \mathcal{X}=\delta\left(\lambda-\lambda^{\prime}\right) \delta_{i j} \\
& \sum_{\lambda, i} \bar{\theta}_{\lambda}^{(i)}(x) \theta_{\lambda}^{(i)}\left(x^{\prime}\right)=\omega^{2-d}(x) \delta \mathcal{X}\left(x, x^{\prime}\right),
\end{aligned}
$$

where the indices $(i),(j)$ label the possible degeneracy of the (possibly continuous) spectrum, $\delta \mathcal{X}\left(x, x^{\prime}\right)$ is the delta distribution on $\mathcal{X}$ and the prefactor $\omega^{2-d}(x)$ comes from the definition of the Hilbert product. 
As in the toy-example treated in the previous section, we introduce "formal quantum fields" $\hat{\varphi}_{\lambda}^{(i)}(y)$ by a smearing out of the above modes:

$$
\hat{\varphi}_{\lambda}^{(i)}(y)=\int_{\mathcal{X}} \widehat{\Phi}(X) \bar{\theta}_{\lambda}^{(i)}(x) \mathrm{d} \tilde{v}_{\mathcal{X}}(x) .
$$

Two remarks are in order here.

First, it is not obvious a priori that this expression makes sense at all, since we are smearing an operator valued distribution with a function which does not belong to the corresponding test function space. At best, the fields $\hat{\varphi}_{\lambda}^{(i)}(y)$ can be operator-valued distributions w.r.t. $\lambda$ and $y$, namely, to get a bona fide operator one should smear $\hat{\varphi}_{\lambda}^{(i)}(y)$ against suitable test functions in $\lambda$ and $y$ (as in the toy-example).

Second, while the Hilbert space $\mathcal{H}$ may seem to be the most natural where to study the eigenvalue problem given in Eq. (22), its choice is by no means mandatory. Different choices may produce different formulæ.

By formally using Eq. (24) we can invert the transformation (25) and get

$$
\widehat{\Phi}(X)=\sum_{\lambda, i} \theta_{\lambda}^{(i)}(x) \hat{\varphi}_{\lambda}^{(i)}(y) .
$$

In concrete applications the actual viability of this inversion needs to be verified case by case.

In the following we use real-valued eigenfunctions $\theta_{\lambda}^{(i)}$ so that the fields $\hat{\varphi}_{\lambda}^{(i)}(y)$ are Hermitean.

Under the assumptions of self-adjointness that we have postulated, the following properties hold:

(a) The fields $\hat{\varphi}_{\lambda}^{(i)}$ satisfy the Klein-Gordon equation on the manifold $\mathcal{Y}$ (in cases of interest to us, $\mathcal{Y}$ is Lorentzian).

(b) The fields $\hat{\varphi}_{\lambda}^{(i)}$ commute for $\lambda \neq \lambda^{\prime}$ or $i \neq j$.

The proof of assertion (a) comes from the following chain of equalities (in the sense of distributions in $\lambda$ and $y$ ):

$$
\begin{aligned}
(\square \mathcal{Y}+\lambda) \hat{\varphi}_{\lambda}^{(i)}(y) & =\int_{\mathcal{X}} \theta_{\lambda}^{(i)}(x)(\square \mathcal{Y}+\lambda) \widehat{\Phi}(X) \mathrm{d} \tilde{v}_{\mathcal{X}}(x) \\
& =\int_{\mathcal{X}} \theta_{\lambda}^{(i)}(x)\left[-\omega^{2}(x)\left(\tilde{\triangle} \mathcal{X}+M^{2}\right)+\lambda\right] \widehat{\Phi}(X) \mathrm{d} \tilde{v} \mathcal{X}(x) \\
& =\int_{\mathcal{X}}\left\{\left[-\omega^{2}(x)\left(\tilde{\triangle} \mathcal{X}+M^{2}\right)+\lambda\right] \theta_{\lambda}^{(i)}(x)\right\} \widehat{\Phi}(X) \mathrm{d} \tilde{v}_{\mathcal{X}}(x)=0,
\end{aligned}
$$

where we made use of the assumed self-adjointness of the operator $\omega^{2}\left(\tilde{\triangle} \mathcal{X}+M^{2}\right)$ (but not of the hermiticity of the fields).

The two-point correlation functions of the fields $\hat{\varphi}_{\lambda}^{(i)}$ on the vacuum $\Omega$ of the field $\widehat{\Phi}$ is then given by: 


$$
\begin{aligned}
W_{\lambda, \lambda^{\prime}}^{(i j)}\left(y, y^{\prime}\right) & =\left\langle\Omega, \hat{\varphi}_{\lambda}^{(i)}(y) \hat{\varphi}_{\lambda^{\prime}}^{(j)}\left(y^{\prime}\right) \Omega\right\rangle \\
& =\int_{\mathcal{X} \times \mathcal{X}} \mathrm{d} \tilde{v}_{\mathcal{X}}(x) \mathrm{d} \tilde{v}_{\mathcal{X}}\left(x^{\prime}\right) \theta_{\lambda}^{(i)}(x) \theta_{\lambda^{\prime}}^{(j)}\left(x^{\prime}\right) W\left(X, X^{\prime}\right) .
\end{aligned}
$$

If we invert this formula by making use of (24), we obtain the following representation for $W$ :

$$
W\left(X, X^{\prime}\right)=\sum_{\lambda, \lambda^{\prime}, i, j} \theta_{\lambda}^{(i)}(x) \theta_{\lambda^{\prime}}^{(j)}\left(x^{\prime}\right) W_{\lambda, \lambda^{\prime}}^{(i j)}\left(y, y^{\prime}\right) .
$$

The distribution $W_{\lambda \lambda^{\prime}}^{(i j)}\left(y, y^{\prime}\right)$ satisfies the Klein-Gordon equation on $\mathcal{Y}$ w.r.t. both $y$ and $y^{\prime}$, with masses $\sqrt{\lambda}$ and respectively $\sqrt{\lambda^{\prime}}$.

We now prove assertion (b), namely that the quantum fields $\hat{\varphi}_{\lambda}^{(i)}$ commute for different values of $\lambda$ or $i$. Indeed, the CCR's for the field $\widehat{\Phi}$ can be written as follows:

$$
\begin{aligned}
{\left[\widehat{\Phi}(X), \widehat{\Phi}\left(X^{\prime}\right)\right]_{L_{\mathcal{C}}} } & =0 \\
{\left[\widehat{\Phi}(X), \partial_{t^{\prime}} \widehat{\Phi}\left(X^{\prime}\right)\right]_{L_{\mathcal{C}}} } & =\mathrm{i} \delta_{\mathcal{C}}\left(X, X^{\prime}\right),
\end{aligned}
$$

where $\partial_{t}$ denotes a time-like vector, orthogonal to a given Cauchy surface $\mathcal{C}$ and normalized to unity (this is not necessarily the gradient of a time parameter). We have adopted the following convention: whenever we have a (Riemannian) submanifold $S \hookrightarrow \mathcal{M}$, then $\delta_{S}\left(p, p^{\prime}\right)$ denotes the delta distribution on that submanifold w.r.t. the volume element inherited from the ambient manifold $\mathcal{M}$.

Taking advantage of the structure of $\mathcal{M}$, we can choose a Cauchy surface in the form $\mathcal{C}=\mathcal{X} \times \Sigma$, where $\Sigma$ is a Cauchy surface in $\mathcal{Y}$; the former equations now read

$$
\begin{aligned}
& {\left[\widehat{\Phi}(X), \widehat{\Phi}\left(X^{\prime}\right)\right]_{L_{\mathcal{C}}}=0,} \\
& {\left[\widehat{\Phi}(X), \partial_{t^{\prime}} \widehat{\Phi}\left(X^{\prime}\right)\right]_{L_{\mathcal{C}}}=\mathrm{i} \delta_{\mathcal{C}}\left(X, X^{\prime}\right)=\mathrm{i} \omega^{1-d}(x) \delta_{\mathcal{X}}\left(x, x^{\prime}\right) \delta_{\Sigma}\left(y, y^{\prime}\right),}
\end{aligned}
$$

the factor $\omega^{1-d}$ comes from the volume element of $\mathcal{C}$ which is given by $\mathrm{d} v_{\mathcal{C}}=\omega^{d-1} \mathrm{~d} v_{\mathcal{X}} \otimes$ $\mathrm{d} v_{\Sigma}$ (recall that the surface $\Sigma$ has dimension $\left.d-1\right)$. The vector $\partial_{t}$ is a time-like vector orthogonal to $\mathcal{C}=\mathcal{X} \times \Sigma$ and normalized w.r.t. the metric of $\mathcal{M}$ : it follows that the vector $\omega(x) \partial_{t}$ is a time-like vector orthogonal to $\Sigma$ and normalized w.r.t. the metric in $\mathcal{Y} .{ }^{1}$ We will denote by $\partial_{T}$ the vector $\omega(x) \partial_{t}$ tangent to $\mathcal{Y}$ and also (with a slight abuse of notation) its lift to the tangent bundle of $\mathcal{M}$. With this rescaling Eq. (33) reads

$$
\left[\widehat{\Phi}(X), \partial_{T^{\prime}} \widehat{\Phi}\left(X^{\prime}\right)\right]_{L \mathcal{C}}=\mathrm{i} \omega(x) \delta_{\mathcal{C}}\left(X, X^{\prime}\right)=\mathrm{i} \omega^{2-d}(x) \delta_{\mathcal{X}}\left(x, x^{\prime}\right) \delta_{\Sigma}\left(y, y^{\prime}\right) .
$$

We now smear both sides of Eqs. (32) and (34) with the modes $\theta_{\lambda}^{(i)}(x)$ and $\theta_{\lambda^{\prime}}^{(j)}\left(x^{\prime}\right)$ and apply Eq. (25): Eq. (32) gives an analogous equation for the fields $\hat{\varphi}_{l}^{(i)}$ and Eq. (34) gives

$$
\left[\hat{\varphi}_{\lambda}^{(i)}(y), \partial_{T^{\prime}} \hat{\varphi}_{\lambda^{\prime}}^{(j)}\left(y^{\prime}\right)\right]_{\lfloor\Sigma}
$$

\footnotetext{
${ }^{1}$ Indeed, let $\partial_{t}$ be a normalized vector tangent to $\mathcal{M}=\mathcal{X} \times \omega \mathcal{Y}$ at the point $(x, y)$ : then its projection onto $\mathcal{Y}$ has norm $\omega^{-2}(x)$, for $1=g\left(\partial_{t}, \partial_{t}\right)=\omega^{2}(x)^{(\mathcal{Y})} g\left(\partial_{t}, \partial_{t}\right)$.
} 


$$
\begin{aligned}
& =\int_{\mathcal{X}} \mathrm{d} \tilde{v}_{\mathcal{X}}(x) \int_{\mathcal{X}} \mathrm{d} \tilde{v}_{\mathcal{X}}\left(x^{\prime}\right) \theta_{\lambda}^{(i)}(x) \theta_{\lambda^{\prime}}^{(j)}\left(x^{\prime}\right) \omega(x)^{2-d} \delta_{\mathcal{X}}\left(x, x^{\prime}\right) \delta_{\Sigma}\left(y, y^{\prime}\right) \\
& =\delta_{i j} \delta\left(\lambda-\lambda^{\prime}\right) \delta_{\Sigma}\left(y, y^{\prime}\right) .
\end{aligned}
$$

It follows that $\hat{\varphi}_{\lambda}^{(i)}$ commutes everywhere on $\mathcal{Y}$ with $\hat{\varphi}_{\lambda^{\prime}}^{(j)}$ for $\lambda \neq \lambda^{\prime}$ or $\lambda=\lambda^{\prime}$ but $i \neq j$ : in fact the above equations tell that on the Cauchy surface $\Sigma$, for $\lambda \neq \lambda^{\prime}$, the Klein-Gordon fields $\hat{\varphi}_{\lambda}, \hat{\varphi}_{\lambda^{\prime}}$ commute between themselves along with their canonical momenta and hence they do commute everywhere in $\mathcal{Y}$ as a consequence of the equations of motion. This ends the proof of assertion (b).

In all the examples that we shall present (as in the toy-example of the previous section), this commutativity of the formal fields will follow from a stronger property, namely the diagonal character of their correlation functions $W_{\lambda, \lambda^{\prime}}^{(i j)}\left(y, y^{\prime}\right)$, which will be of the form $\delta_{i j} \delta\left(\lambda-\lambda^{\prime}\right) W_{\lambda}\left(y, y^{\prime}\right)$. This stronger property may fail to be true in the generic case, unless some additional structural properties on $\mathcal{M}$ are introduced. This is precisely what will be done in our next section, in such a way that all our examples are covered .

Whenever the previous diagonal form of $W_{\lambda, \lambda^{\prime}}^{(i j)}\left(y, y^{\prime}\right)$ is valid, Eq. (29) immediately yields the corresponding diagonal decomposition:

$$
\left\langle\Omega, \widehat{\Phi}(X) \widehat{\Phi}\left(X^{\prime}\right) \Omega\right\rangle=\sum_{\lambda, i} \theta_{\lambda}^{(i)}(x) \theta_{\lambda}^{(i)}\left(x^{\prime}\right) W_{\lambda}\left(y, y^{\prime}\right) .
$$

Moreover, when we consider the field $\widehat{\Phi}$ restricted to a fixed slice $x=$ const, we obtain a superposition of Klein-Gordon fields as an immediate consequence of the previous formula, namely:

$$
\left\langle\Omega, \widehat{\Phi}(x, y) \widehat{\Phi}\left(x, y^{\prime}\right) \Omega\right\rangle=\sum_{\lambda, i}\left|\theta_{\lambda}^{(i)}(x)\right|^{2} W_{\lambda}\left(y, y^{\prime}\right) .
$$

This formula is analogous to the Källen-Lehmann representation for the two-point function in the Minkowskian spacetime [17].

From (37) it follows that the weight function of this Källen-Lehmann decomposition of the restricted propagator is:

$$
\mu^{(i)}(\lambda, x)=\sum_{\lambda^{\prime}, j} \delta_{i j} \delta\left(\lambda-\lambda^{\prime}\right)\left|\theta_{\lambda^{\prime}}^{(j)}(x)\right|^{2},
$$

which is the discontinuity of the resolvent of the operator $\omega^{2}(x)\left(\tilde{\Delta}_{\mathcal{X}}+M^{2}\right)$ on its spectrum, i.e., the density of states per unit spectrum per unit volume (in $\mathcal{X}$ ).

If $\mathcal{X}$ is a one-dimensional spatial manifold we may take one step further.

Let us choose a coordinate $x$ such that the line element on $\mathcal{X}$ is simply $-\mathrm{d} x^{2}$. The spectral problem now leads to

$$
\omega^{2}(x)\left(\varphi^{\prime \prime}(x)+d \frac{\omega^{\prime}(x)}{\omega(x)} \varphi^{\prime}(x)-M^{2} \varphi(x)\right)=-\lambda \varphi(x),
$$

where the Hilbert space has the inner product

$$
(\varphi, \psi)=\int_{\mathcal{X}} \mathrm{d} x \omega^{d-2}(x) \bar{\varphi}(x) \psi(x) .
$$


The transformation

$$
\varphi(x)=\omega^{\frac{1-d}{2}}(x) f(x)
$$

allows us to rewrite the eigenvalue equation and the Hilbert product as follows:

$$
\begin{aligned}
& f^{\prime \prime}(x)+\frac{\omega^{\prime}(x)}{\omega(x)} f^{\prime}(x)+ {\left[\frac{\lambda}{\omega^{2}(x)}-M^{2}+\frac{1-d}{2} \frac{\omega^{\prime \prime}(x)}{\omega(x)}\right.} \\
&\left.-\frac{(d-1)^{2}}{4}\left(\frac{\omega^{\prime}(x)}{\omega(x)}\right)^{2}\right] f(x)=0, \\
&(f, h)=\int_{\mathcal{X}} \frac{\mathrm{d} x}{\omega(x)} \bar{f}(x) h(x) .
\end{aligned}
$$

Let us introduce a coordinate $s$ so that

$$
\mathrm{d} s=\frac{\mathrm{d} x}{\omega(x)} .
$$

We obtain that:

$$
\begin{aligned}
& -f^{\prime \prime}(s)+U(s) f(s)=\lambda f(s), \\
& (f, h)=\int_{\mathcal{X}} \bar{f}(s) h(s) \mathrm{d} s,
\end{aligned}
$$

where

$$
U(s)=\frac{d-1}{2} \frac{\omega^{\prime \prime}(s)}{\omega(s)}+\left(\frac{\omega^{\prime}(s)}{\omega(s)}\right)^{2}\left[\frac{(d-1)^{2}}{4}+\frac{1-d}{2} \omega(s)\right]+M^{2} \omega^{2}(s),
$$

and prime now means derivative w.r.t. the variable $s$.

We have obtained a one-dimensional Schrödinger problem with a potential $U(s)$ which depends on the warping function $\omega(s)$. Notice that the result matches the introductory example for the flat case; this is a trivial instance of the above general framework, where $\mathcal{X}=\mathbb{R}, \mathcal{Y}=\mathbb{R}^{d}$ and $\omega(x)=1$ : the operator $\omega^{2}(x)\left(\tilde{\triangle} \mathcal{X}+M^{2}\right)=-\partial_{x}^{2}+M^{2}$ describes exactly a free Schrödinger particle with constant potential $M^{2}$.

\section{Warped manifolds with additional geometrical structure}

In order to give relevant applications of the previous theoretical setting, we need to specify additional structural properties on the geometry of the warped manifold $\mathcal{M}$. These geometrical properties will be sufficient to establish (via the lemma stated below) the validity of the diagonal decomposition (36) which then entails the existence of a KällenLehmann-type decomposition for the bulk Klein-Gordon fields built in terms of a "tower" of massive fields living on the brane $\mathcal{Y}$.

Such geometrical properties involve appropriate consistency requirements between the geometry of $\mathcal{M}$ and that of the leaves $\mathcal{Y}_{x}$ that deal with global symmetries as well as with the existence of complexified manifolds for $\mathcal{M}$ and $\mathcal{Y}$. 
(i) Consistency of global isometries of the leaves

We assume that there exists an isometry group $G$ of $\mathcal{M}$ and a subgroup ${ }^{2} G \mathcal{Y}$ of $G$ acting in each leaf $\mathcal{Y}_{x}$ of $\mathcal{M}$ as a global isometry group of $\mathcal{Y}$ and that there exists a global pseudo-distance $z\left(y, y^{\prime}\right)$ on $\mathcal{Y}$ which is preserved by this isometry group $G \mathcal{Y}$.

(ii) Consistency of complex geometries

We assume that $\mathcal{M}$ and $\mathcal{Y}$ admit respective complexified manifolds $\mathcal{M}^{(c)}$ and $\mathcal{Y}^{(c)}$, such that for each $x$ in $\mathcal{X}$ the complexified $\mathcal{Y}_{x}^{(c)}$ of $\mathcal{Y}_{x}$ is contained in $\mathcal{M}^{(c)}$. Moreover, $\mathcal{M}^{(c)}$ and $\mathcal{Y}^{(c)}$ contain distinguished pairs of domains, called respectively the tuboids $T^{ \pm}$and $\mathcal{T}^{ \pm}$in such a way that for all $x$ in $\mathcal{X}$, one has:

$$
\mathcal{T}_{x}^{+} \subset T^{+} \text {and } \mathcal{T}_{x}^{-} \subset T^{-} .
$$

These tuboids $T^{ \pm}$(resp. $\mathcal{T}^{ \pm}$) serve to define a preferred class of (generalized) free fields on $\mathcal{M}$ (resp. $\mathcal{Y}$ ), as being those whose two-point functions are boundary values of holomorphic functions $\mathrm{W}\left(X, X^{\prime}\right)$ (resp. $\left.\mathrm{W}\left(y, y^{\prime}\right)\right)$ in the product domain $T^{-} \times T^{+}$(resp. $\mathcal{T}^{-} \times \mathcal{T}^{+}$). This property, which is a generalization of the standard analyticity property of Minkowskian two-point functions in the Wightman axiomatic framework, is called normal analyticity (see its introduction in the de Sitter case in [2] and more recently its extension to the anti-de Sitter case in [19]).

On the basis of the previous consistency requirements, we shall now establish the following statement (where we have kept the notations of the previous section, but dropped the discrete variables $i, j)$ :

Lemma. Consider the distribution in $\left(\lambda, \lambda^{\prime}\right)$ defined as the two-point function of the formal fields $\hat{\varphi}_{\lambda}(y)$ and $\hat{\varphi}_{\lambda^{\prime}}\left(y^{\prime}\right)$, namely

$$
\begin{aligned}
W_{\lambda, \lambda^{\prime}}\left(y, y^{\prime}\right) & =\left\langle\Omega, \hat{\varphi}_{\lambda}(y) \hat{\varphi}_{\lambda^{\prime}}\left(y^{\prime}\right) \Omega\right\rangle \\
& =\int_{\mathcal{X} \times \mathcal{X}} \mathrm{d} \tilde{v}_{\mathcal{X}}(x) \mathrm{d} \tilde{v} \mathcal{X}\left(x^{\prime}\right) \theta_{\lambda}(x) \theta_{\lambda^{\prime}}\left(x^{\prime}\right) W\left(X, X^{\prime}\right),
\end{aligned}
$$

where $W\left(X, X^{\prime}\right)$ denotes the two-point function of a Klein-Gordon field $\widehat{\Phi}(X)$ on $\mathcal{M}$ satisfying $G$-invariance and normal analyticity in $\mathcal{M}^{(c)}$, and the integral over $x$ and $x^{\prime}$ in (47) is supposed to be convergent after smearing out in the variables $\lambda, \lambda^{\prime}$ for all real or complex values of $y$ and $y^{\prime}\left(\right.$ in $\left.\mathcal{T}^{-} \times \mathcal{T}^{+}\right)$.

Then the distribution $W_{\lambda, \lambda^{\prime}}\left(y, y^{\prime}\right)$ is of the following diagonal form

$$
W_{\lambda, \lambda^{\prime}}\left(y, y^{\prime}\right)=\delta\left(\lambda-\lambda^{\prime}\right) W_{\lambda}\left(y, y^{\prime}\right)
$$

where $W_{\lambda}\left(y, y^{\prime}\right)=w_{\lambda}\left(z\left(y, y^{\prime}\right)\right)$ is a solution of the Klein-Gordon equation (in both variables $\left.y, y^{\prime}\right)$

$$
\square_{y} W_{\lambda}\left(y, y^{\prime}\right)=\square_{y^{\prime}} W_{\lambda}\left(y, y^{\prime}\right)=-\lambda W_{\lambda}\left(y, y^{\prime}\right)
$$

\footnotetext{
${ }^{2}$ It is not necessary that $G$ is a global isometry group of $\mathcal{M} ; G$ can be identical to $G \mathcal{Y}$, as it will occur in most of the applications below. However, in the latter there will be a larger global isometry group acting on an extension $\widehat{\mathcal{M}}$ of $\mathcal{M}$ on which the ambient two-point function $W\left(X, X^{\prime}\right)$ is defined and admits this global isometry.
} 
satisfying the required properties of a two-point function on $\mathcal{Y}$, namely $G$ y-invariance and normal analyticity property in $\mathcal{Y}^{(c)}$. Moreover, $W_{\lambda}\left(y, y^{\prime}\right)$ is correctly normalized, in consistency with the canonical commutation relation for the corresponding Klein-Gordon field, namely, one has (with the notations of Section 2):

$$
\partial_{T^{\prime}}\left(W_{\lambda}\left(y, y^{\prime}\right)-W_{\lambda}\left(y^{\prime}, y\right)\right)_{L_{\Sigma}}=\delta_{\Sigma}\left(y, y^{\prime}\right) .
$$

To show this lemma we observe that, in view of (47), the invariance of $W\left(X, X^{\prime}\right)$ under $G$ implies the invariance of $W_{\lambda, \lambda^{\prime}}\left(y, y^{\prime}\right)$ under $G \mathcal{Y}$. The latter is therefore of the form $W_{\lambda, \lambda^{\prime}}\left(y, y^{\prime}\right)=w_{\lambda, \lambda^{\prime}}\left(z\left(y, y^{\prime}\right)\right)$ and in view of the symmetry of the distance w.r.t. $y$ and $y^{\prime}$, one has in the sense of distributions in $\left(\lambda, \lambda^{\prime}\right)$ :

$$
\square_{y} W_{\lambda, \lambda^{\prime}}\left(y, y^{\prime}\right)-\square_{y^{\prime}} W_{\lambda, \lambda^{\prime}}\left(y, y^{\prime}\right)=0
$$

and therefore, in view of property (a) of the fields $\hat{\varphi}_{\lambda}(y)$ :

$$
\left(\lambda-\lambda^{\prime}\right) W_{\lambda, \lambda^{\prime}}\left(y, y^{\prime}\right)=0
$$

which entails that $W_{\lambda, \lambda^{\prime}}\left(y, y^{\prime}\right)$ is of the form (48) (since the general solution as a distribution of the equation $x_{1} T\left(x_{1}, x_{2}\right)=0$ is $\left.T\left(x_{1}, x_{2}\right)=\delta\left(x_{1}\right) \times t\left(x_{2}\right)\right)$. The normal analyticity of $W_{\lambda}\left(y, y^{\prime}\right)$ results from the normal analyticity of $W\left(X, X^{\prime}\right)$ in view of the inclusion relations (46) and the assumed convergence of the integral in (47). Finally, the normalization of $W_{\lambda}\left(y, y^{\prime}\right)$ readily follows from the commutation relation (35) established in Section 2 by integrating the latter over $\lambda^{\prime}$.

\section{Applications}

In the four examples studied below, we discuss quantum field theories on manifolds which admit natural complexified manifolds carrying tuboids of normal analyticity, and in all these theories the geometric symmetries are unbroken, namely the considered two-point functions $W\left(X, X^{\prime}\right)$ are invariant under the global isometries of the ambient manifold $\mathcal{M}$; moreover, the leaves $\mathcal{Y}_{x}$ will always satisfy the two geometrical consistency requirements specified above.

In the first two examples, $\mathcal{Y}$ is a de Sitter spacetime and $\mathcal{X}$ will be the half line or the segment $(0, \pi)$ with appropriate measures; they give a structure of warped product to open subsets of the Minkowski space in the first example, and to an ambient de Sitter spacetime in the second example: this extends and generalizes the results in [11].

In the third example we will revisit the Unruh problem, namely the restriction of an ambient Minkowskian quantum field theory to the world-line $\mathcal{Y}$ of a uniformly accelerated observer. In this case, the isometry group of $\mathcal{Y}$ (induced by a Lorentz boost subgroup of the ambient space) is simply the time translation group in the proper time of the accelerated observer.

The last example regards QFT on the anti-de Sitter manifold, considered as foliated by Minkowskian branes. Although this case seems to lie out of the picture drawn in Section 2, because AdS spacetime is not globally hyperbolic, it turns out that this lack of global 
hyperbolicity is not an obstruction to the applicability of the previous lemma. In fact, the previous geometrical consistency requirements are still fulfilled there.

In all these examples, the diagonal form (48) of the correlators $W_{\lambda, \lambda^{\prime}}^{(i j)}\left(y, y^{\prime}\right)$ of the formal fields $\hat{\varphi}_{\lambda}^{(i)}, \hat{\varphi}_{\lambda^{\prime}}^{(j)}$ will always allow us to interpret each formal field $\hat{\varphi}_{\lambda}^{(i)}$ as a genuine KleinGordon field with the corresponding two-point function $W_{\lambda}\left(y, y^{\prime}\right)$ on the brane, and to obtain thereby, via the inversion argument given in Section 2 (based on the completeness relation (24)), a decomposition of the ambient Wightman function $W\left(X, X^{\prime}\right)$ with the diagonal form (36).

The consistency requirements which we consider in this section readily imply (without any computation) that the restriction to any given leaf $\mathcal{Y}_{x}$ of any Klein-Gordon field of the ambient space $\mathcal{M}$ is a generalized free field on this leaf. When the branes are either Minkowski or de Sitter spacetimes, as in the examples we will present, there exists also a direct method for computing the spectral function of this restricted field by a Laplacetype transformation on the leaf (this is standard for the Wightman fields in Minkowski space [7] and has been carried out for de Sitter fields in [2] by using the results on "invariant perikernels on the one-sheeted hyperboloids" of [23]). It is to be expected that the comparison of such Laplace-type expressions of the spectral function with the one obtained here by the (completely different and more general) warped-manifold method in terms of the "Schrödinger modes" $\theta_{\lambda}^{i}$ will provide new interesting identities relating Hankel-type and Legendre-type functions.

Concerning the more technical problem of the convergence of the (a priori formal) integrals and sums (47) and (36), we shall check the latter in all the examples and prove in particular that (36) can be given a well-defined meaning as an integral w.r.t. a suitable measure over the allowed mass spectrum and possibly a sum over the degeneracy indices. To this end we shall analyze the spectral problem along the general lines drawn in Section 2.

In the first three examples the operator $T_{\mathcal{X}}=\omega^{2}(x)\left(\widetilde{\triangle}_{\mathcal{X}}+M^{2}\right)$ is essentially selfadjoint in $L^{2}\left(\mathcal{X}, d \tilde{v}_{\mathcal{X}}\right)$ on the domain $C_{0}^{\infty}(\mathcal{X})$ because it reduces to ordinary Schrödinger operators with smooth potentials bounded from below; therefore we will not discuss their self-adjointness, since this follows from general theorems (see, e.g., [20]).

On the contrary, in the last anti-de Sitter example the relevant operator is essentially selfadjoint only for values of $M^{2}$ bigger than a certain threshold $M_{0}^{2}$; below $M_{0}^{2}$ the operator is not essentially self-adjoint but can be extended to a self adjoint operator in many different ways. Among the infinite a priori allowable extensions, two of them are of special relevance to the so-called AdS-CFT correspondence [19].

\subsection{Decomposition of (bulk) Minkowski fields into de Sitter (brane) fields}

In this example the manifold $\mathcal{M}$ is the set of all points which are space-like w.r.t. a given event, chosen as the origin in a $(d+1)$-dimensional Minkowski spacetime, endowed with a system of inertial coordinates denoted by $\left\{X^{\mu}\right\}, \mu=0, \ldots, d$.

The region $\mathcal{M}=\left\{X: X^{\mu} X_{\mu}<0\right\}$ is foliated by a family of $d$-dimensional de Sitter spacetimes, identified with the hyperboloids 


$$
X \cdot X \equiv \eta_{\mu \nu} X^{\mu} X^{\nu}=\left(X^{0}\right)^{2}-(\vec{X})^{2}=-R^{2} .
$$

$\mathcal{M}$ has the structure of a warped manifold with base $\mathcal{X}=\mathbb{R}^{+}$with coordinate $R$; the fiber $\mathcal{Y}$ can be identified with a $d$-dimensional de Sitter spacetime with radius $R=1$; using a polar-like parametrization for the events of $\mathcal{M}, X=R y$ with $y^{2}=-1$, the Minkowskian metric of $\mathcal{M}$ can then be rewritten as follows:

$$
\mathrm{d} s^{2}=-\mathrm{d} R^{2}+R^{2} \mathrm{~d} s_{\mathcal{Y}}^{2},
$$

where $\mathrm{d} s_{\mathcal{Y}}^{2}$ is the de Sitter metric of $\mathcal{Y}$, obtained as restriction of the Minkowski metric of the ambient space. This realizes $\mathcal{M}$ as a warped product with warping function $\omega(R)=R$.

The operator $\tilde{\Delta}_{\mathcal{X}}$ equals $-\partial_{R}^{2}-\frac{\mathrm{d}}{R} \partial_{R}$ and we are led to the following eigenvalue equation for the modes $\theta_{\lambda}$ :

$$
R^{2}\left(-\partial_{R}^{2}-\frac{d}{R} \partial_{R}+M^{2}\right) \theta_{\lambda}(R)=\lambda \theta_{\lambda}(R) .
$$

The operator at the 1.h.s. is essentially self-adjoint on the dense domain $\mathcal{C}_{0}^{\infty}$ of the Hilbert space $L^{2}\left(\mathcal{X}, d \tilde{v}_{\mathcal{X}}\right)$, whose scalar product has the following explicit form:

$$
(\varphi, \psi)=\int_{\mathcal{X}} \bar{\varphi}(R) \psi(R) R^{d-2} \mathrm{~d} R .
$$

By means of the transformation (41) and by rescaling $\rho=M R$ (which together are particular instances of the so called "Lommel's transformations"), Eq. (49) is turned into the modified Bessel's equation. By further introducing the new variable $M R=\mathrm{e}^{s}$ we finally obtain:

$$
-f_{\lambda}^{\prime \prime}+\left(\mathrm{e}^{2 s}-v^{2}\right) f_{\lambda}=0
$$

with

$$
v^{2}=\lambda-\frac{(d-1)^{2}}{4} .
$$

The prime now means derivatives w.r.t. the variable $s$. This operator is now self-adjoint w.r.t. the standard $L^{2}$ product $\int_{\mathbb{R}} \bar{f}(s) h(s) \mathrm{d} s$.

We have thus obtained a Schrödinger problem for a potential $\mathrm{e}^{2 s}$. The corresponding spectrum is absolutely continuous and nondegenerate; it coincides with the positive real line. This implies the condition $\lambda>(d-1)^{2} / 4$.

The solutions which have the correct asymptotic behavior at $s=\infty$ are $K_{i v}\left(\mathrm{e}^{s}\right)$, where $K_{i v}(z)=K_{-i v}(z)$ denotes the modified Bessel function [24]; it is real for real $v$. The normalization can be obtained by studying the asymptotic behavior at $s=-\infty$, where these solutions behave as free waves.

The final result, expressed in the original coordinate $R$, is the following family of normalized generalized eigenfunctions:

$$
\begin{aligned}
& \theta_{\lambda}(R)=N_{\lambda} R^{\frac{1-d}{2}} K_{i \sqrt{\lambda-(d-1)^{2} / 4}}(M R), \\
& N_{\lambda} \equiv \frac{1}{\pi} \sqrt{\sinh \left(\pi \sqrt{\lambda-(d-1)^{2} / 4}\right)} .
\end{aligned}
$$


There hold the completeness and orthonormality relations:

$$
\begin{aligned}
& \int_{\frac{(d-1)^{2}}{4}}^{\infty} \mathrm{d} \lambda \theta_{\lambda}(R) \theta_{\lambda}\left(R^{\prime}\right)=R^{-(d-2)} \delta\left(R-R^{\prime}\right), \\
& \int_{\mathbb{R}^{+}} \mathrm{d} R R^{d-2} \theta_{\lambda}(R) \theta_{\lambda^{\prime}}(R)=\delta\left(\lambda-\lambda^{\prime}\right) .
\end{aligned}
$$

We now introduce the fields $\hat{\varphi}_{\lambda}(y)$ on the de Sitter manifold $\mathcal{Y}$ by smearing the field $\widehat{\Phi}$ against the radial modes (53), as in Eq. (25). The main result of this section is the following:

(c) The fields $\hat{\varphi}_{\lambda}(y)$ correspond to de Sitter Klein-Gordon fields in the "Euclidean" [1] (also called Bunch-Davies [25]) vacuum state, namely the vacuum expectation value (v.e.v.) of $\hat{\varphi}_{\lambda}(y)$ on $\mathcal{Y}$ is given by

$$
W_{\lambda, \lambda^{\prime}}\left(y, y^{\prime}\right)=\left\langle\Omega\left|\hat{\varphi}_{\lambda}(y) \hat{\varphi}_{\lambda^{\prime}}\left(y^{\prime}\right)\right| \Omega\right\rangle=\delta\left(\lambda-\lambda^{\prime}\right) W_{\lambda}^{(E, d)}\left(y, y^{\prime}\right),
$$

where $W_{\lambda}^{(E, d)}$ is the d-dimensional Euclidean (Bunch-Davies) two-point function, equipped with its normal analytic structure [2]. Moreover, each Klein-Gordon field of the ambient Minkowski space (with arbitrary positive mass $M$ ) admits the following expansion of its two-point function:

$$
\left\langle\Omega, \widehat{\Phi}(X) \widehat{\Phi}\left(X^{\prime}\right) \Omega\right\rangle=\int_{(d-1)^{2} / 4}^{\infty} \mathrm{d} \lambda \theta_{\lambda}(R) \theta_{\lambda}\left(R^{\prime}\right) W_{\lambda}^{(E, d)}\left(y, y^{\prime}\right),
$$

with $\theta_{\lambda}(R)$ given by formula (53).

This equation allows us to consider the quantum field $\widehat{\Phi}$ restricted to a fixed de Sitter brane $R=R^{\prime}$; it has the structure of a Källen-Lehmann expansion expressing the ambient quantum field $\widehat{\Phi}$ as a superposition of de Sitter quantum fields on the brane $\hat{\varphi}_{\lambda}$ with mass spectrum

$$
\left\langle\Omega, \widehat{\Phi}(X) \widehat{\Phi}\left(X^{\prime}\right) \Omega\right\rangle_{\left.\right|_{R=R^{\prime}}}=\int_{(d-1)^{2} / 4}^{\infty} \mathrm{d} \lambda\left|\theta_{\lambda}(R)\right|^{2} W_{\lambda}^{(E, d)}\left(y, y^{\prime}\right) .
$$

The proof of the previous statement goes as follows: according to [2], both geometrical consistency requirements defined above are satisfied by the subset $\mathcal{M}$ of Minkowski space and the de Sitter leaf $\mathcal{Y}$ : the isometry group $G=G \mathcal{Y}$ is the corresponding Lorentz group $S O_{0}(1, d)$ and the tubes $\mathcal{T}_{R}^{ \pm}$are the intersections of the complex quadric $\mathcal{Y}_{R}^{(c)}$ with the tubes $T^{ \pm}$of the complexified Minkowski space $\mathcal{M}^{(c)}=\mathbf{C}^{d+1}$. It follows that the previous lemma is applicable, and that we only have to check that the two-point function $W_{\lambda}\left(y, y^{\prime}\right)$ of formula (48) coincides in the present case with the function $W_{\lambda}^{(E, d)}$. Let us recall that $W_{\lambda}^{(E, d)}$ is a distribution in the de Sitter invariant variable $v=y \cdot y^{\prime}$ which satisfies the de Sitterian Klein-Gordon equation with eigenvalue $-\lambda$ in both variables $y, y^{\prime}$ and is given by

$$
W_{\lambda}^{(E, d)}\left(y, y^{\prime}\right)=C_{d, v} P_{-\frac{d-1}{2}+\mathrm{i} v}^{(d+1)}\left(y \cdot y^{\prime}\right)
$$


where

$$
\begin{aligned}
& C_{d, v}=\frac{\Gamma((d-1) / 2+\mathrm{i} v) \Gamma((d-1) / 2-\mathrm{i} v)}{2^{d} \Gamma(d / 2) \pi^{d / 2}}, \\
& P_{-\frac{d-1}{2}+\mathrm{i} v}^{(d+1)}(v)=2^{\frac{d-2}{2}} \Gamma(d / 2)\left(v^{2}-1\right)^{\frac{2-d}{4}} P_{-\frac{1}{2}-\mathrm{i} v}^{\frac{2-d}{2}}(v),
\end{aligned}
$$

and $P_{b}^{a}$ denotes the associated Legendre function [24].

The value of the constant $C_{d, v}$ ensures the correct normalization of $W_{\lambda}^{(E, d)}$, the canonical commutation relations being satisfied by the corresponding Klein-Gordon field. Moreover this distribution is correctly defined as being the boundary value of the holomorphic function $P_{-\frac{d-1}{2}+\mathrm{iv}}^{(d+1)}\left(y \cdot y^{\prime}\right)$ from the tuboid $\left\{\left(y, y^{\prime}\right) \in \mathcal{T}^{-} \times \mathcal{T}^{+}\right\}$of $\mathcal{Y}^{(c)} \times \mathcal{Y}^{(c)}$ [2].

So by all its properties, this definition of $W_{\lambda}^{(E, d)}$ coincides with that of $W_{\lambda}\left(y, y^{\prime}\right)$ given in the lemma, which proves formula (55), and therefore the rest of property (c) (in view of (24)).

It is worthwhile to remark that, when explicitly written Eq. (56) is a rather complicated new integral relation between Legendre and Hankel functions. Here we get a "quantum field theoretical" proof of that integral relation without actually performing any integral.

It is interesting to derive an alternative expression for $W_{\lambda, \lambda^{\prime}}\left(y, y^{\prime}\right)$ by plugging the momentum representation of the Minkowskian two-point function $W\left(X, X^{\prime}\right)$ into its defining formula (47). We obtain:

$$
\begin{aligned}
W_{\lambda, \lambda^{\prime}}\left(y, y^{\prime}\right)= & \int_{0}^{\infty} \frac{\mathrm{d} R}{R} R^{d-1} \theta_{\lambda}(R) \int_{0}^{\infty} \frac{\mathrm{d} R^{\prime}}{R^{\prime}} R^{\prime d-1} \theta_{\lambda^{\prime}}\left(R^{\prime}\right) \\
& \times \int \frac{\mathrm{d}^{d+1} P}{(2 \pi)^{d}} \delta\left(P^{2}-M^{2}\right) \Theta\left(P_{0}\right) \mathrm{e}^{-\mathrm{i} P\left(X-X^{\prime}\right)} .
\end{aligned}
$$

In this expression we insert the parametrizations $X=R y$ and $X^{\prime}=R^{\prime} y^{\prime}$ and introduce a vector $\alpha$ defined by the relation $M \alpha=P$, so that $\alpha$ varies on the unit shell. One then rewrites the subintegral over $P$ as

$$
\int \frac{\mathrm{d}^{d+1} \alpha}{(2 \pi)^{d}} \delta\left(\alpha^{2}-1\right) \Theta\left(\alpha_{0}\right) \mathrm{e}^{\mathrm{i}\left(y^{\prime}-y\right) \cdot \alpha M R} .
$$

and by exchanging the order of the integrations over $R, R^{\prime}$ and $\alpha$ one is led to introduce the following integrals:

$$
\begin{aligned}
\varphi_{\lambda}(y, \alpha)= & \varphi_{\lambda}(y \cdot \alpha)=M^{\frac{d-1}{2}} \int_{0}^{\infty} \mathrm{e}^{\mathrm{i} y \cdot \alpha M R} \theta_{\lambda}(R) R^{d-1} \frac{\mathrm{d} R}{R} \\
= & \sqrt{\frac{\pi}{2}} N_{\lambda} \Gamma((d-1) / 2-\mathrm{i} v) \Gamma((d-1) / 2+\mathrm{i} v) \\
& \times\left((-\mathrm{i} y \cdot \alpha)^{2}-1\right)^{\frac{2-d}{4}} P_{-\frac{1}{2}-\mathrm{i} v}^{\frac{2-d}{2}}(-\mathrm{i} y \cdot \alpha) .
\end{aligned}
$$

The functions $\varphi_{\lambda}(y, \alpha)$ are plane waves on de Sitter manifold, i.e., are modes satisfying the de Sitter Klein-Gordon equation whose phase is constant on planes; $\alpha$ plays the role 
of wave-vector. $P$ is an associated Legendre function [24]. The integral appearing in the definition of these waves is well defined at both extrema provided $|\Im(v)|<\frac{d-1}{2}$.

Then by rewriting the integral (60) in terms of these waves, we obtain the following new integral representation for the Bunch-Davies de Sitter two-point function:

$$
\begin{aligned}
W_{\lambda, \lambda^{\prime}}\left(y, y^{\prime}\right) & =\delta\left(\lambda-\lambda^{\prime}\right) C_{d, v} P_{-\frac{d-1}{2}+i v}^{(d+1)}\left(y \cdot y^{\prime}\right) \\
& =\int \frac{\mathrm{d}^{d+1} \alpha}{(2 \pi)^{d}} \delta\left(\alpha^{2}-1\right) \Theta\left(\alpha_{0}\right) \bar{\varphi}_{\lambda}(y \cdot \alpha) \varphi_{\lambda^{\prime}}\left(y^{\prime} \cdot \alpha\right) .
\end{aligned}
$$

\subsection{Decomposition of (bulk) de Sitter fields into lower dimensional (brane) de Sitter fields}

In the second example we are dealing with a family of $d$-dimensional de Sitter branes embedded in a $(d+1)$-dimensional de Sitter spacetime. As explained in [27] this problem is physically relevant to understand the spectrum of the density fluctuations in an open inflationary cosmology.

Let us consider a $(d+2)$-dimensional Minkowski spacetime, with a chosen set of inertial coordinates $X^{0}, \ldots, X^{d+1}$. The bulk de Sitter manifold is taken to be the unit one-sheeted hyperboloid $D S=\left\{X \in \mathbb{M}^{d+2}, X \cdot X=-1\right\}$. Consider now the following open region of the bulk: $\left\{X \in \mathrm{DS}:\left|X^{d+1}\right|<1\right\}$. This region is foliated by $d$-dimensional de Sitter branes, obtained by intersecting the bulk with a family of hyperplanes parameterized by a coordinate $x \in(0, \pi)$ as follows: $\left\{X \in \mathbb{M}^{d+2}, X^{d+1}=\cos x\right\}$.

The metric of the bulk de Sitter manifold can consequently be written as follows:

$$
\mathrm{d} s_{\mathrm{DS}}^{2}=-\mathrm{d} x^{2}+\sin ^{2} x \mathrm{~d} s_{\mathrm{dS}}^{2} ;
$$

$\mathrm{d} s_{\mathrm{dS}}^{2}$ is the metric of a $d$-dimensional de Sitter manifold with radius $R=1$, and $\omega(x)=$ $\sin x$. The base manifold $\mathcal{X}$ is thus the segment $(0, \pi)$ with coordinate $x$ and metric $\mathrm{d} x^{2}$.

The spectral problem now is the following:

$$
\omega^{2}(x)\left(\tilde{\triangle}_{\mathcal{X}}+M^{2}\right) \theta_{\lambda}=\left(\sin ^{2} x\right) \theta_{\lambda}^{\prime \prime}+d\left(\cos ^{2} x\right) \theta_{\lambda}^{\prime}-\left(\sin ^{2} x\right) M^{2} \theta_{\lambda}=-\lambda \theta_{\lambda} ;
$$

it has to be considered in the Hilbert space whose product is $(\varphi, \psi)=\int_{0}^{\pi}(\sin x)^{d-2} \bar{\varphi}(x)$ $\psi(x) \mathrm{d} x . M$ is the mass of the field propagating in the ambient de Sitter space.

Following Eq. (41), this equation can be simplified by introducing $\theta(x)=\sin ^{\frac{(1-d)}{2}} x f(x)$. A further simplification is achieved by introducing the coordinate $s=\operatorname{arctanh} \cos x$. The operator and the inner product become

$$
\begin{aligned}
& -f^{\prime \prime}(s)+\frac{M^{2}-\frac{d^{2}-1}{4}}{\cosh ^{2}(s)} f(s)=\left(\lambda-\frac{(d-1)^{2}}{4}\right) f(s), \\
& (f, h)=\int_{\mathbb{R}} \mathrm{d} s \bar{f}(s) h(s),
\end{aligned}
$$

where again the prime denotes the derivative w.r.t. $s$. We have obtained a Schrödinger problem with potential $U(s)=\frac{M^{2}-\frac{d^{2}-1}{4}}{\cosh ^{2}(s)}$ : this is either a barrier or a well according to 
the sign of $M^{2}-\frac{d^{2}-1}{4}$. When this quantity is negative some bound states may appear depending on the depth of the well. In both cases a positive continuous doubly-degenerate spectrum will persist, for which $\lambda-\frac{(d-1)^{2}}{4}=q^{2} \geqslant 0$. It is now a standard quantum mechanical problem to find eigenfunctions and eigenvalues of this Schrodinger problem.

\section{Continuous spectrum}

The continuous spectrum coincides with the positive real axis. We will write $q^{2}=$ $\lambda-\frac{(d-1)^{2}}{4}$ with $\lambda>\frac{(d-1)^{2}}{4}$ for the eigenvalue. Using standard techniques for the study of Schrödinger operators one can find the following family of orthonormal complex generalized eigenfunctions labeled by a positive parameter $q$ :

$$
\begin{aligned}
& F_{q}(x)=\frac{\mathrm{e}^{\frac{\pi q}{2}} \Gamma(1+\rho-\mathrm{i} q) \Gamma(-\rho-\mathrm{i} q)}{\sqrt{2 \pi} \Gamma(-\mathrm{i} q)}(\sin x)^{\frac{1-d}{2}} P_{\rho}^{i q}(\cos x+\mathrm{i} \epsilon), \\
& F_{-q}=\frac{\mathrm{e}^{\frac{-\pi q}{2}} \Gamma(1+\rho-\mathrm{i} q) \Gamma(-\rho-\mathrm{i} q)}{\sqrt{2 \pi} \Gamma(-\mathrm{i} q)}(\sin x)^{\frac{1-d}{2}} P_{\rho}^{i q}(-\cos x-\mathrm{i} \epsilon),
\end{aligned}
$$

where $\rho$ satisfies

$$
\rho(\rho+1)=\frac{d^{2}-1}{4}-M^{2}=-\frac{1}{4}-v^{2}
$$

(see Eq. (59)) so that $\rho=-\frac{1}{2}+\mathrm{i} \nu$. The required real modes $\theta_{q, \epsilon}$ with $\epsilon \in\{s, c\}$ are given by

$$
\begin{aligned}
& \theta_{q, s}(x)=\frac{1}{2 \mathrm{i}}\left(F_{q}(x)-\overline{F_{q}(x)}\right), \\
& \theta_{q, c}(x)=\frac{1}{2}\left(F_{q}(x)+\overline{F_{q}(x)}\right) .
\end{aligned}
$$

\section{Discrete spectrum}

When $M^{2}-\frac{d^{2}-1}{4}<0$, bound states can exist. We can construct them by the substitution $q \rightarrow-i q$ in the formulæ for the generalized eigenfunctions corresponding to the continuous spectrum; now $\rho$ is solution of

$$
(\rho+1) \rho=\frac{d^{2}-1}{4}-M^{2} \text {. }
$$

We fix the root $\rho=-\frac{1}{2}+\sqrt{\frac{d^{2}}{4}-M^{2}}>0$. Standard quantum mechanics then says that the discrete eigenvalues are

$$
q_{n}=-n-\frac{1}{2}+\sqrt{\frac{d^{2}}{4}-M^{2}}>0 .
$$

Consequently, the number of bound states is

$$
\#\{\text { Discrete spectrum }\}=\left[-\frac{1}{2}+\sqrt{\frac{d^{2}}{4}-M^{2}}\right],
$$


where the square brackets denote the integral part. The normalization of the corresponding states can be computed using the following integral [26]

$$
\int_{-1}^{1} \frac{\mathrm{d} y}{1-y^{2}}\left|P_{\rho}^{-q}(y)\right|^{2}=\frac{\Gamma(1+\rho-q)}{q \Gamma(1+\rho+q)} .
$$

It follows that the normalized eigenfunctions are

$$
\theta_{n}(x)=(\sin x)^{\frac{1-d}{2}} P_{\rho}^{-\rho+n}(\cos x+\mathrm{i} \epsilon) \sqrt{\frac{(\rho-n) \Gamma(1+2 \rho-n)}{n !}},
$$

with $n=0, \ldots,[\rho]=\left[-\frac{1}{2}+\sqrt{\frac{d^{2}}{4}-M^{2}}\right]$. As before, let us introduce the formal quantum fields

$$
\begin{aligned}
& \hat{\varphi}_{q, \epsilon}(y)=\int_{0}^{\pi} \mathrm{d} x(\sin x)^{d-2} \theta_{q, \epsilon}(x) \widehat{\Phi}(x, y), \quad q \in \mathbb{R}^{+}, \epsilon \in\{s, c\}, \\
& \hat{\varphi}_{n}(y)=\int_{0}^{\pi} \mathrm{d} x(\sin x)^{d-2} \theta_{n}(x) \widehat{\Phi}(x, y) .
\end{aligned}
$$

By the same arguments used in Section 4.1 we obtain that:

(d) The fields $\hat{\varphi}_{q, \epsilon}(y), \hat{\varphi}_{n}$ are Klein-Gordon fields on de Sitter brane in the Euclidean vacuum state, namely their ambient de Sitter v.e.v. in the $(d+1)$-dimensional Euclidean vacuum is given by

$$
\begin{aligned}
& W_{\lambda, \epsilon ; \lambda^{\prime}, \epsilon^{\prime}}\left(y, y^{\prime}\right)=\left\langle\Omega\left|\hat{\varphi}_{q, \epsilon}(y) \hat{\varphi}_{q^{\prime}, \epsilon^{\prime}}\left(y^{\prime}\right)\right| \Omega\right\rangle=\delta\left(\lambda-\lambda^{\prime}\right) \delta_{\epsilon \epsilon^{\prime}} W_{\lambda}^{(E, d)}\left(y, y^{\prime}\right), \\
& W_{n ; n^{\prime}}\left(y, y^{\prime}\right)=\left\langle\Omega\left|\hat{\varphi}_{n}(y) \hat{\varphi}_{n^{\prime}}\left(y^{\prime}\right)\right| \Omega\right\rangle=\delta_{n n^{\prime}} W_{\lambda_{n}}^{(E, d)}\left(y, y^{\prime}\right) .
\end{aligned}
$$

All other correlators vanish identically.

Here $W_{\lambda}^{(E, d)}$ is the Euclidean two-point function in $d$ dimensions (see Eq. (58)) with square mass $\lambda$. By inverting now the completeness relations for the fields

$$
\widehat{\Phi}(X)=\sum_{n} \theta_{n}(x) \hat{\varphi}_{n}(y)+\sum_{\epsilon} \int_{\mathbb{R}} \mathrm{d} q \theta_{q, \epsilon}(x) \hat{\varphi}_{q, \epsilon}(y)
$$

we obtain the following decomposition of the Euclidean de Sitter two-point function in terms of lower dimensional ones; this is quite a nontrivial relation between Legendre functions in different dimensions:

$$
\begin{aligned}
& W_{M}^{(E, d+1)}\left(X, X^{\prime}\right)=\sum_{n=0}^{[\rho]} \theta_{n}(x) \theta_{n}\left(x^{\prime}\right) W_{\lambda_{n}}^{(E, d)}\left(y, y^{\prime}\right) \\
& \quad+\sum_{\epsilon} \int_{(d-1)^{2} / 4}^{\infty} \mathrm{d} \lambda \theta_{\left(\lambda-(d-1)^{2} / 4\right)^{1 / 2}, \epsilon}(x) \theta_{\left(\lambda-(d-1)^{2} / 4\right)^{1 / 2}, \epsilon}\left(x^{\prime}\right) W_{\lambda}^{(E, d)}\left(y, y^{\prime}\right)
\end{aligned}
$$




$$
\begin{aligned}
& =\sum_{n=0}^{[\rho]} \overline{P_{\rho}^{-\rho+n}\left(\cos x^{\prime}+\mathrm{i} \epsilon\right)} P_{\rho}^{-\rho+n}(\cos x+\mathrm{i} \epsilon) \\
& \times \frac{(\rho-n) \Gamma(1+2 \rho-n)}{n !\left(\sin x \sin x^{\prime}\right)^{(d-1) / 2}} W_{\lambda_{n}}^{(E, d)}\left(y, y^{\prime}\right) \\
& +\int_{\mathbb{R}} \frac{\mathrm{d} q \sinh (\pi q) q}{2 \pi^{2}\left(\sin x \sin x^{\prime}\right)^{(d-1) / 2}}|\Gamma(1+\rho-\mathrm{i} q) \Gamma(-\rho-\mathrm{i} q)|^{2} \\
& \times\left[\mathrm{e}^{\pi q} \overline{P_{\rho}^{i q}\left(\cos x^{\prime}+\mathrm{i} \epsilon\right)} P_{\rho}^{i q}(\cos x+\mathrm{i} \epsilon)\right. \\
& \left.+\mathrm{e}^{-\pi q} \overline{P_{\rho}^{i q}\left(-\cos x^{\prime}-\mathrm{i} \epsilon\right)} P_{\rho}^{i q}(-\cos x-\mathrm{i} \epsilon)\right] W_{q^{2}+\frac{(d-1)^{2}}{4}}^{(E, d)}\left(y, y^{\prime}\right) .
\end{aligned}
$$

On a fixed de Sitter brane $x=x^{\prime}$ we get a Källen-Lehmann type decomposition of the correlator of the bulk quantum field, with a measure given by

$$
\begin{aligned}
\mu(q, x)= & \sum_{0}^{[\rho]}\left|P_{\rho}^{-\rho+n}(\cos x+\mathrm{i} \epsilon)\right|^{2} \frac{(\rho-n) \Gamma(1+2 \rho-n)}{n ! \sin ^{d-1}(x)} \delta(q-(\rho-n)) \\
& +\frac{\sinh (\pi q) q}{2 \pi^{2} \sin ^{d-1}(x)}|\Gamma(1+\rho-\mathrm{i} q) \Gamma(-\rho-\mathrm{i} q)|^{2} \\
& \times\left[\mathrm{e}^{\pi q}\left|P_{\rho}^{i q}(\cos x+\mathrm{i} \epsilon)\right|^{2}+\mathrm{e}^{-\pi q}\left|P_{\rho}^{i q}(-\cos x-\mathrm{i} \epsilon)\right|^{2}\right] .
\end{aligned}
$$

In all these formulæ the discrete contribution vanishes whenever $M^{2} \geqslant\left(\frac{d-1}{2}\right)^{2}$.

We remark that the formula of this decomposition matches the one in [27] which was obtained by the completely different method of Laplace-type transform.

\subsection{Decomposition of Minkowski states into uniformly accelerated world-lines (Unruh effect)}

In this section we revisit the Unruh effect; the general framework is the same as in the previous examples, except that now the codimension of the leaves $\mathcal{Y}$ is maximal (i.e., $d$, where the dimension of the ambient manifold is $d+1$ ). What is new in the present approach to this old model, is that we obtain a closed formula for the decomposition of the ambient QFT into a collection of harmonic oscillators which oscillate in the proper time of the accelerated observer and not in the time of an inertial observer. Now the ambient manifold is the wedge $\mathcal{M}=\left\{X \in \mathbb{M}^{d+1}:\left|X^{0}\right|<X^{d}\right\}$ of a Minkowskian spacetime $\mathbb{M}^{d+1}$ and $\mathcal{Y}$ is the unidimensional world-line of an accelerated observer. In this case, the field $\widehat{\Phi}$ will be reduced to a set of harmonic oscillators.

An uniformly accelerated world-line is conveniently parametrized by

$$
(\xi \sinh \tau, \vec{x}, \xi \cosh \tau),
$$

where $\vec{x}$ are the remaining $d-1$ coordinates in Minkowski space. In terms of these coordinates the wedge acquires the structure of warped product of a $d$-dimensional Riemannian half space $\mathcal{X}=\mathbb{R}_{+}^{d}$ with a 1-dimensional timelike line $\mathcal{Y}$, with warping function $\omega(\xi, \vec{x})=\xi$ : 


$$
\mathrm{d} s^{2}=\xi^{2} \mathrm{~d} \tau^{2}-\mathrm{d} \xi^{2}-\sum_{1}^{d-1}\left(\mathrm{~d} x^{i}\right)^{2}
$$

The transverse problem is

$$
\xi^{2}\left(\tilde{\triangle} \mathcal{X}+M^{2}\right) \theta(\xi, \vec{x})=\xi^{2}\left[-\partial_{\xi}^{2}-\sum_{1}^{d-1}\left(\frac{\partial}{\partial x^{i}}\right)^{2}-\frac{1}{\xi} \partial_{\xi}+M^{2}\right] \theta(\xi, \vec{x})=\lambda \theta,
$$

and the corresponding Hilbert product

$$
(\varphi, \psi)=\int_{\mathbb{R}_{+}^{d}} \frac{\mathrm{d} \xi}{\xi}\left(\prod_{1}^{d-1} \mathrm{~d} x^{i}\right) \bar{\varphi}(\xi, \vec{x}) \psi(\xi, \vec{x})
$$

A straightforward computation produces the following generalized orthonormal eigenfunctions

$$
\begin{aligned}
\theta_{\lambda}(\xi, \vec{x}) & =\theta_{m, \vec{p}, \pm}(\xi, \vec{x})=\frac{\sqrt{2 \sinh (\pi m)}}{\pi} K_{i m}\left(\xi \sqrt{M^{2}+\vec{p}^{2}}\right) \frac{1}{(2 \pi)^{\frac{d-1}{2}}}\left\{\begin{array}{c}
\cos (\vec{p} \cdot \vec{x}) \\
\sin (\vec{p} \cdot \vec{x})
\end{array}\right\} \\
& =N_{m} K_{i m}\left(\xi \sqrt{M^{2}+\vec{p}^{2}}\right)\left\{\begin{array}{c}
\cos (\vec{p} \cdot \vec{x}) \\
\sin (\vec{p} \cdot \vec{x})
\end{array}\right\},
\end{aligned}
$$

where the \pm subscript selects among $\cos (\vec{p} \cdot \vec{x})$ and $\sin (\vec{p} \cdot \vec{x})$. In this case the eigenvalue $\lambda=m^{2}$ has a $\mathbb{R}^{d-1}$ degeneracy. Again, we introduce the quantum fields

$$
\hat{\varphi}_{\lambda, \vec{p}, \pm}(\tau)=\int_{0}^{\infty} \frac{\mathrm{d} \xi}{\xi} \int_{\mathbb{R}^{d-2}} \mathrm{~d} \vec{x} \theta_{\lambda, \vec{p}, \pm}(\xi, \vec{x}) \widehat{\Phi}(\tau, \xi, \vec{x}) .
$$

Let now $W\left(X, X^{\prime}\right)$ be the usual Wightman two-point function for the quantum field $\widehat{\Phi}(X)$ as given by Eq. (3): we can directly compute the correlators $W_{m, \vec{p}, \epsilon ; m^{\prime} \vec{p}^{\prime}, \epsilon^{\prime}}\left(\tau, \tau^{\prime}\right)$ of the fields $\hat{\varphi}_{\lambda, \vec{p}, \pm}(\tau)$ and show that they are diagonal in $m, \vec{p}$ and the discrete index $\epsilon \in\{+,-\}$. Indeed

$$
\begin{array}{r}
W_{m, \vec{p}, \epsilon ; m^{\prime}, \vec{p}^{\prime}, \epsilon^{\prime}}\left(\tau, \tau^{\prime}\right)=\int_{\mathbb{R}_{+}^{d}} \frac{\mathrm{d} \xi}{\xi} \mathrm{d} \vec{x} \int_{\mathbb{R}_{+}^{d}} \frac{\mathrm{d} \xi^{\prime}}{\xi^{\prime}} \mathrm{d} \vec{x}^{\prime} \theta_{m, \vec{p}, \epsilon}(\xi, \vec{x}) \theta_{m^{\prime}, \vec{p}^{\prime}, \epsilon^{\prime}}\left(\xi^{\prime}, \vec{x}^{\prime}\right) W\left(X, X^{\prime}\right) \\
=\int_{\mathbb{R}_{+}^{d}} \frac{\mathrm{d} \xi}{\xi} \mathrm{d} \vec{x} \int_{\mathbb{R}_{+}^{d}} \frac{\mathrm{d} \xi^{\prime}}{\xi^{\prime}} \mathrm{d} \vec{x}^{\prime} N_{m} K_{i m}\left(\xi \sqrt{M^{2}+\vec{p}^{2}}\right)\left\{\begin{array}{l}
\cos (\vec{p} \cdot \vec{x}) \\
\sin (\vec{p} \cdot \vec{x})
\end{array}\right\} \\
\times N_{m^{\prime}} K_{i m^{\prime}}\left(\xi^{\prime} \sqrt{M^{2}+\vec{p}^{\prime 2}}\right)\left\{\begin{array}{l}
\cos \left(\vec{p}^{\prime} \cdot \vec{x}\right) \\
\sin \left(\vec{p}^{\prime} \cdot \vec{x}\right)
\end{array}\right\} \frac{1}{(2 \pi)^{d+1}}, \\
\int_{\mathbb{R}^{d+1} \frac{\mathrm{d}^{d+1} P}{(2 \pi)^{d}} \delta\left(P^{2}-M^{2}\right) \Theta\left(P_{0}\right) \mathrm{e}^{\mathrm{i} P \cdot\left(X-X^{\prime}\right)}} \\
=N_{m} N_{m^{\prime}} \int_{\mathbb{R}_{+}} \frac{\mathrm{d} \xi}{\xi} \int_{\mathbb{R}_{+}} \frac{\mathrm{d} \xi^{\prime}}{\xi^{\prime}} K_{i m}\left(\xi \sqrt{M^{2}+\vec{p}^{2}}\right) K_{i m^{\prime}}\left(\xi^{\prime} \sqrt{M^{2}+\vec{p}^{\prime 2}}\right) \delta\left(\vec{p}-\vec{p}^{\prime}\right) \delta_{\epsilon, \epsilon^{\prime}}
\end{array}
$$




$$
\begin{aligned}
& \int_{\mathbb{R}^{2}} \frac{\mathrm{d} P_{0} \mathrm{~d} P_{1}}{2 \pi} \delta\left(P_{0}^{2}-P_{1}^{2}-\vec{p}^{2}-M^{2}\right) \Theta\left(P_{0}\right) \\
& \quad \times \mathrm{e}^{\mathrm{i} P_{0}\left(\xi \sinh (\tau)-\xi^{\prime} \sinh \left(\tau^{\prime}\right)\right)+\mathrm{i} P_{1}\left(\xi \cosh (\tau)-\xi^{\prime} \cosh \left(\tau^{\prime}\right)\right)} .
\end{aligned}
$$

The remaining integration is a special case of Eq. (60) with the substitutions $M^{2} \mapsto$ $M^{2}+\vec{p}^{2}, v \mapsto m, d=1$. This finally gives

$$
W_{m, \vec{p}, \epsilon ; m^{\prime}, \vec{p}^{\prime}, \epsilon^{\prime}}\left(\tau, \tau^{\prime}\right)=\delta\left(\vec{p}-\vec{p}^{\prime}\right) \delta_{\epsilon, \epsilon^{\prime}} \delta\left(m^{2}-m^{\prime 2}\right) \frac{\cos \left(m\left(\tau-\tau^{\prime}\right)+\mathrm{i} \pi m\right)}{2 m \sinh (\pi m)} .
$$

This expression is the Wightman function of an harmonic oscillator in a thermal state at an inverse temperature $\beta$ (in the Heisenberg picture): indeed, the quantum Klein-Gordon field on a one-dimensional spacetime corresponds to a single quantum harmonic oscillator in the Heisenberg picture where the mass represents the spring constant. The thermal time correlation function of the position operator at inverse temperature $\beta$ for such oscillator is given by:

$$
W\left(t, t^{\prime}\right)=\frac{\cos \left(\omega\left(t-t^{\prime}+\mathrm{i} \beta / 2\right)\right)}{2 \omega \sinh (\omega \beta / 2)},
$$

which is precisely the expression derived above with $\beta=2 \pi$.

Using the completeness of the modes $\theta$ we can express the vacuum two-point function of the field $\widehat{\Phi}$ in terms of the two-point functions of the thermal oscillators as in

$$
\begin{aligned}
W\left(X, X^{\prime}\right)= & \sum_{\epsilon=+,-} \int_{\mathbb{R}^{d-1}} \mathrm{~d} \vec{p} \int_{0}^{\infty} \mathrm{d}\left(m^{2}\right) \theta_{m, \vec{p}, \epsilon}(\xi, \vec{x}) \theta_{m, \vec{p}, \epsilon}\left(\xi^{\prime}, \vec{x}^{\prime}\right) \\
& \times \frac{\cos \left(m\left(\tau-\tau^{\prime}\right)+\mathrm{i} \pi m\right)}{2 m \sinh (\pi m)} .
\end{aligned}
$$

In this case we know that if the state of the ambient field $\Phi(X)$ is the usual vacuum one, the quantum theory obtained from the ambient space one is thermal at the Unruh inverse temperature $\beta_{U}=2 \pi$. The decomposition

$$
\left\langle\Omega, \Phi(\tau, \xi, \vec{x}) \Phi\left(\tau^{\prime}, \xi, \vec{x}\right) \Omega\right\rangle=\int \mu((\xi, \vec{x}), m)\left\langle\hat{\varphi}_{m}(\tau) \hat{\varphi}_{m}\left(\tau^{\prime}\right)\right\rangle_{\beta_{U}}
$$

defines correlation functions $\left\langle\varphi_{m}(\tau) \varphi_{m}\left(\tau^{\prime}\right)\right\rangle_{\beta_{U}}$ of a thermal state of the quantum harmonic oscillator given by $\frac{\mathrm{d}^{2}}{\mathrm{~d} \tau^{2}} \varphi_{m}(\tau)+m^{2} \varphi^{2}(\tau)=0$. Note that along each uniformly accelerated world-line, specified by the parameters $\xi$ and $\vec{x}$, the corresponding proper-time is equal to $\xi \tau$ ( $\xi$ being the value of the Tolman factor), so that the temperature "really felt by the corresponding observer" on this world-line is equal to $1 /(2 \pi \xi)$.

\subsection{AdS states in terms of Minkowski states}

This last example concerns the states of a Klein-Gordon field theory on the AdS spacetime foliated by flat Minkowski spacetimes of codimension one: this decomposition has been used in [19] in application to the AdS-CFT correspondence and it will be just briefly reported. 
This example lies somewhat outside of the picture we have drawn in the general part because the AdS spacetime is not globally hyperbolic. Nevertheless we can prove directly that a completely analogous decomposition of the Klein-Gordon field can be achieved.

To set the notation, in the spirit of Section 4.2 we consider the vector space $\mathbb{R}^{d+2}$ equipped with the following pseudo-scalar product:

$$
X \cdot X^{\prime}=X^{0}{X^{\prime}}^{0}-X^{1} X^{\prime 1}-\cdots-X^{d}{X^{\prime}}^{d}+X^{d+1} X^{\prime d+1} .
$$

The $(d+1)$-dimensional AdS universe can then be identified with the quadric

$$
A d S_{d+1}=\left\{X \in \mathbb{R}^{d+2}, X^{2}=R^{2}\right\},
$$

where $X^{2}=X \cdot X$, endowed with the induced metric

$$
\mathrm{d} s_{A d S}^{2}=\left.\left(\mathrm{d}\left(X^{0}\right)^{2}-\mathrm{d}\left(X^{1}\right)^{2}-\cdots+\mathrm{d}\left(X^{d+1}\right)^{2}\right)\right|_{A d S_{d+1}} .
$$

The AdS relativity group is $G=S O_{0}(2, d)$, that is the connected component of the identity of the pseudo-orthogonal group $S O(2, d)$. Two events $X, X^{\prime}$ of $A d S_{d+1}$ are space-like separated if $\left(X-X^{\prime}\right)^{2}<0$, i.e., if $X \cdot X^{\prime}>R^{2}$. In the following we will put for notational simplicity $R=1$.

We consider an open subset of AdS given by the inequality in the ambient space $\Pi \equiv$ $\left\{X^{d}+X^{d+1}>0\right\}$ : this is "half" the spacetime. In the "horocyclic parametrization" $X=$ $X(x, y)$, there appears a structure of warped product: this set of coordinates covers $\Pi$ and is obtained by intersecting $A d S_{d+1}$ with the hyperplanes $\left\{X^{d}+X^{d+1}=\mathrm{e}^{x}=\frac{1}{s}\right\}$ each slice $\Pi_{v}$ (or "horosphere") being an hyperbolic paraboloid:

$$
\left\{\begin{array}{l}
X^{\mu}=\mathrm{e}^{x} y^{\mu}=\frac{1}{s} y^{\mu}, \quad \mu=0,1, \ldots, d-1, \\
X^{d}=\sinh x+\frac{1}{2} \mathrm{e}^{x} y^{2}=\frac{1-s^{2}}{2 s}+\frac{1}{2 s} y^{2}, \quad y^{2}=y^{0^{2}}-y^{1^{2}}-\cdots-y^{d-1^{2}}, \\
X^{d+1}=\cosh x-\frac{1}{2} \mathrm{e}^{x} y^{2}=\frac{1+s^{2}}{2 s}-\frac{1}{2 s} y^{2} .
\end{array}\right.
$$

In each slice $\Pi_{v}, y^{0}, \ldots, y^{d-1}$ can be seen as coordinates of an event of a $d$-dimensional Minkowski spacetime $\mathbb{M}^{d}$ with metric $\mathrm{d} s_{M}^{2}=\mathrm{d}\left(y^{0}\right)^{2}-\mathrm{d}\left(y^{1}\right)^{2}-\cdots-\mathrm{d}\left(y^{d-1}\right)^{2}$ (here and in the following where it appears, an index $M$ stands for Minkowski). This explains why the horocyclic coordinates $(x, y)$ of the parametrization (85) are also called Poincaré coordinates. The scalar product (85) and the AdS metric can then be rewritten as follows:

$$
\begin{aligned}
& X \cdot X^{\prime}=\cosh \left(x-x^{\prime}\right)-\frac{1}{2} \mathrm{e}^{x+x^{\prime}}\left(y-y^{\prime}\right)^{2}, \\
& \mathrm{~d} \sigma_{A d S}^{2}=\mathrm{e}^{2 x} \mathrm{~d} \sigma_{M}^{2}-\mathrm{d} x^{2}=\frac{1}{s^{2}}\left(\mathrm{~d} \sigma_{M}^{2}-\mathrm{d} s^{2}\right) .
\end{aligned}
$$

Eq. (87) exhibits the region $\Pi$ of $A d S_{d+1}$ as a warped product with warping function $\omega(x)=\mathrm{e}^{x}$ and fibers conformal to $\mathbb{M}^{d}$.

We apply the formalism of Section 2 and obtain the spectral problem

$$
\mathrm{e}^{2 x}\left[\theta^{\prime \prime}(x)+\mathrm{d} \theta^{\prime}(x)-M^{2} \theta(x)\right]=-\lambda \theta(x),
$$

to be considered in the Hilbert space $L^{2}\left(\mathbb{R}, \mathrm{e}^{(d-2) x} \mathrm{~d} x\right)$, where the differential operator defined in Eq. (88) is symmetric. In the variable $s=\mathrm{e}^{-x}$ already introduced in Eq. (85) 
and defining $f(s)=\theta(x) \mathrm{e}^{\frac{d-1}{2} x}$ Eq. (88) is turned into the well-known Schrödinger spectral problem on the half-line

$$
-f^{\prime \prime}(s)+\frac{M^{2}+\frac{d^{2}-1}{4}}{s^{2}} f(s)=-f^{\prime \prime}(s)+\frac{(v+1 / 2)(v-1 / 2)}{s^{2}} f(s)=\lambda f(s) .
$$

Following [28, p. $88 \mathrm{ff}$.], we learn that there are two distinct regimes corresponding to the two ranges $v \geqslant 1$ and $|v|<1$.

When $v \geqslant 1$ the previous operator is essentially self-adjoint and there is only one possible choice for the generalized eigenfunctions, namely

$$
f_{\lambda}(s)=\frac{1}{\sqrt{2}} s^{1 / 2} J_{v}(\sqrt{\lambda} s),
$$

where $J_{v}$ are Bessel's functions. The completeness of these eigenfunctions gives Hankel's formula, which expresses the resolution of the identity in $L^{2}\left(\mathbb{R}^{+}, \mathrm{d} s\right)$ as follows:

$$
g(s)=\int_{0}^{\infty} \mathrm{d} \lambda f_{\lambda}(s) \int_{0}^{\infty} f_{\lambda}\left(s^{\prime}\right) g\left(s^{\prime}\right) \mathrm{d} s^{\prime}, \forall g \in L^{2}\left(\mathbb{R}^{+}, \mathrm{d} s\right) .
$$

When $0 \leqslant v<1$ both solutions $s^{1 / 2} J_{v}(\sqrt{\lambda} s)$ and $s^{1 / 2} J_{-v}(\sqrt{\lambda} s)$ are square integrable in the neighborhood of $s=0$ and must be taken into consideration: we are in the so-called limit circle case at zero [20,28], which implies that the operator is not essentially selfadjoint and there exists a $S^{1}$ ambiguity in the self-adjoint extensions we can perform. The freedom is exactly in the choice of the boundary conditions at $s=0$ (corresponding to the boundary of AdS).

Now we have a one-parameter family of eigenfunctions:

$$
f_{\lambda}^{(\varkappa)}(s) \equiv \sqrt{\frac{s}{2}}\left(\varkappa^{2}-2 \varkappa \lambda^{v} \cos (\pi v)+\lambda^{2 v}\right)^{-1 / 2}\left[\varkappa J_{v}(\sqrt{\lambda} s)-\lambda^{v} J_{-v}(\sqrt{\lambda} s)\right],
$$

to which we must add one bound state when $\varkappa>0$ :

$$
f_{\text {bound }}^{(\varkappa)}(s) \equiv \sqrt{2 \varkappa^{1 / v} \frac{\sin \pi v}{\pi v}} s^{1 / 2} K_{v}\left(\varkappa^{1 / 2 v} s\right) .
$$

The possible choices of the parameter $\varkappa$ do correspond to different self-adjoint extensions of the differential operator (89). To each such extension there is associated a domain $\mathfrak{D}^{(\varkappa)}$ also depending on the parameter $\varkappa$ [20]. To construct $\mathfrak{D}^{(\varkappa)}$ consider the one dimensional subspaces $H_{ \pm}$spanned by the eigenfunctions solving Eq. (89) with eigenvalues $\pm \mathrm{i}$ :

$$
f_{ \pm}(s) \equiv \sqrt{s} K_{v}\left(\mathrm{e}^{ \pm \frac{\mathrm{i} \pi}{4}} s\right)
$$

both these functions are square-integrable when $0 \leqslant v<1$. Each extension is in one-to-one correspondence with partial isometries $U: H_{+} \mapsto H_{-}$, namely, in this case, with elements of $U(1) \simeq S^{1}$. The domain of the extension is obtained by adjoining to the original domain of symmetry the subspace $\left(\mathrm{id}_{H_{+}}+U\right) H_{+}$: here it means that we have to add the span of the $L^{2}$ element

$$
f_{\alpha}(s) \equiv f_{+}(s)+\mathrm{e}^{\mathrm{i} \alpha} f_{-}(s) .
$$

which has in our case the asymptotics 


$$
f_{\alpha}(s) \simeq \frac{\pi}{2 \sin (\pi v)}\left[\frac{2^{v}\left(\mathrm{e}^{-\frac{\mathrm{i} \pi v}{4}}+\mathrm{e}^{\mathrm{i} \alpha+\frac{\mathrm{i} \pi v}{4}}\right)}{\Gamma(1-v)} s^{-v}-\frac{2^{-v}\left(\mathrm{e}^{\frac{\mathrm{i} \pi v}{4}}+\mathrm{e}^{\mathrm{i} \alpha-\frac{\mathrm{i} \pi v}{4}}\right)}{\Gamma(1+v)} s^{\nu}\right] .
$$

The generalized eigenfunctions of the operator (89) corresponding to a specific extension have the following asymptotics

$$
\begin{aligned}
f_{\lambda}^{(\varkappa)}(s) \simeq & 2^{-1 / 2} s^{1 / 2}\left(\varkappa^{2}-2 \varkappa \lambda^{v} \cos (\pi v)+\lambda^{2 v}\right)^{-1 / 2} \lambda^{\nu / 2} \\
& \times\left[\varkappa \frac{2^{-v} s^{v}}{\Gamma(1+v)}-\frac{2^{v} s^{-v}}{\Gamma(1-v)}\right] .
\end{aligned}
$$

As usual these functions do not belong to $L^{2}\left(\mathbb{R}^{+}, \mathrm{d} s\right)$ but any wave-packet does; moreover any such wave packet has this asymptotics. This allows us to find which parameter $\varkappa$ corresponds to which unitary operator $\mathrm{e}^{\mathrm{i} \alpha}: H_{+} \mapsto H_{-}$, i.e., to a specific self-adjoint extension. Indeed, by matching the asymptotics in Eqs. (95) with that in Eq. (96) we obtain

$$
\varkappa=\frac{\cos \left(\frac{\alpha}{2}-\frac{\pi v}{4}\right)}{\cos \left(\frac{\alpha}{2}+\frac{\pi v}{4}\right)} .
$$

We consider now a very specific QFT on the AdS spacetime: this QFT is a generalized free field theory which satisfies certain analyticity properties [30]. It depends on the single (complexified) invariant $\zeta=Z \cdot Z^{\prime}=\cosh \left(x-x^{\prime}\right)-\frac{1}{2} \mathrm{e}^{x+x^{\prime}}\left(z-z^{\prime}\right)^{2}$, where now $z$ (respectively, $z^{\prime}$ ) belongs to the complexified Minkowski space and its imaginary part lies in the interior of the future (resp. past) light cone.

Such a QFT is characterized by the $S O(2, d)$-invariant two-point function given by

$$
W_{\nu}^{d+1}\left(Z, Z^{\prime}\right)=w_{\nu}(\zeta)=\frac{\mathrm{e}^{-\mathrm{i} \pi \frac{d-1}{2}}}{(2 \pi)^{\frac{d+1}{2}}}\left(\zeta^{2}-1\right)^{-\frac{d-1}{4}} Q_{\nu-\frac{1}{2}}^{\frac{d-1}{2}}(\zeta) .
$$

The analyticity domains advocated in [30] are such that the complex variable $\zeta$ belongs to the complex plane cut along the segment from -1 to 1 (the "causal cut"). The analogous invariant variable in the Minkowskian case is $\delta=-\left(z-z^{\prime}\right)^{2}$ and the causal cut in this case is the negative real axis: the "Euclidean regime" corresponds to positive real values of $\delta$. We can now show by direct computation that the two-point function (97) in $A d S_{d+1}$ in the whole range $v \in(-1, \infty)$ can be decomposed as follows:

$$
\begin{aligned}
& W_{\nu}^{d+1}\left(Z(x, z), Z^{\prime}\left(x^{\prime}, z^{\prime}\right)\right)=\int_{0}^{\infty} \mathrm{d} \lambda \theta_{\lambda}(x) \theta_{\lambda}\left(x^{\prime}\right) W_{\lambda}^{M, d}\left(z, z^{\prime}\right), \quad v \in[1, \infty), \\
& W_{\nu}^{d+1}\left(Z(x, z), Z^{\prime}\left(x^{\prime}, z^{\prime}\right)\right)=\int_{0}^{\infty} \mathrm{d} \lambda \theta_{\lambda}^{(\infty)}(x) \theta_{\lambda}^{(\infty)}\left(x^{\prime}\right) W_{\lambda}^{M, d}\left(z, z^{\prime}\right), \quad v \in[0,1), \\
& W_{\nu}^{d+1}\left(Z(x, z), Z^{\prime}\left(x^{\prime}, z^{\prime}\right)\right)=\int_{0}^{\infty} \mathrm{d} \lambda \theta_{\lambda}^{(0)}(x) \theta_{\lambda}^{(0)}\left(v^{\prime}\right) W_{\lambda}^{M, d}\left(z, z^{\prime}\right), \quad v \in(-1,0),
\end{aligned}
$$

where $W_{\lambda}^{M, d}\left(z, z^{\prime}\right)$ is the usual two-point function for a Klein-Gordon field on $\mathbb{M}^{d}$ of square mass $\lambda$ in the Wightman vacuum: 


$$
\begin{aligned}
W_{\lambda}^{M, d}\left(z, z^{\prime}\right) & \equiv \int \frac{\mathrm{d}^{d} p}{(2 \pi)^{d-1}} \delta\left(p^{2}-\lambda\right) \Theta\left(p_{0}\right) \mathrm{e}^{-\mathrm{i} p \cdot\left(z-z^{\prime}\right)} \\
& =(2 \pi)^{-d / 2}\left(\frac{\delta}{\sqrt{\lambda}}\right)^{\frac{2-d}{2}} K_{\frac{d-2}{2}}(\sqrt{\lambda} \delta), \quad \delta \equiv-\left(z-z^{\prime}\right)^{2} .
\end{aligned}
$$

In Eqs. (98) the functions $\theta_{\lambda}^{(\infty)}$ and the $\theta_{\lambda}^{(0)}$ belong to the domains of self-adjointness corresponding to the values $\varkappa=\infty$ and $\varkappa=0$ respectively. They explicitly read

$$
\begin{aligned}
\theta_{\lambda}^{(\infty)}(x) & =\frac{1}{\sqrt{2}} \mathrm{e}^{-\frac{d}{2} x} J_{\nu}\left(\sqrt{\lambda} \mathrm{e}^{-x}\right), \\
\theta_{\lambda}^{(0)}(x) & =\frac{1}{\sqrt{2}} \mathrm{e}^{-\frac{d}{2} x} J_{-|v|}\left(\sqrt{\lambda} \mathrm{e}^{-x}\right) .
\end{aligned}
$$

The reason why we must use different self-adjoint extensions is that $W_{v}^{d+1}(Z(x, z)$, $Z\left(x^{\prime}, z^{\prime}\right)$ ), as a function of $x$ (or $\left.x^{\prime}\right)$ belongs to $\mathfrak{D}^{(\infty)}$ when $v \in[0,1)$ while it belongs to $\mathfrak{D}^{(0)}$ when $v \in(-1,0)$ : this can be proved directly by studying the asymptotics.

The three Eqs. (98) are thus summarized into the following formula valid for the whole range of parameter $v$ :

$$
\begin{aligned}
& W_{v}^{d+1}\left(Z(x, z), Z^{\prime}\left(x^{\prime}, z^{\prime}\right)\right) \\
& =(2 \pi)^{-d / 2}\left(s s^{\prime}\right)^{d / 2} \int_{0}^{\infty} \frac{\mathrm{d} \lambda}{2} \lambda^{\frac{d-2}{4}} J_{v}(\sqrt{\lambda} s) J_{v}\left(\sqrt{\lambda} s^{\prime}\right) \delta^{\frac{2-d}{2}} K_{\frac{d-2}{2}}(\sqrt{\lambda} \delta),
\end{aligned}
$$

with, again, $s=\mathrm{e}^{-x}$.

The proof is an application of formula (12) p. 64 in [29], which is the Hankel's transform of the product of two Bessel's functions (we simply adapt the notation)

$$
\begin{aligned}
& \int_{0}^{\infty} \mathrm{d} m m^{\mu+1 / 2} J_{v}(m s) J_{v}\left(m s^{\prime}\right) K_{\mu}(m \delta)\left(m s^{\prime}\right)^{1 / 2} \\
& =\frac{\delta^{\mu} s^{-\mu-1}\left(s^{\prime}\right)^{-\mu-\frac{1}{2}} \mathrm{e}^{-\left(\mu+\frac{1}{2}\right) \mathrm{i} \pi}}{\sqrt{2 \pi}}\left(\zeta^{2}-1\right)^{-\frac{\mu}{2}-\frac{1}{4}} Q_{\nu-\frac{1}{2}}^{\mu+\frac{1}{2}}(\zeta), \\
& \Re(v)>-1, \quad \Re(\mu+v)>-1,
\end{aligned}
$$

where $\zeta=\frac{s^{2}+\left(s^{\prime}\right)^{2}+\delta^{2}}{2 s s^{\prime}}$. Here we implicitly perform the "Wick rotation" to the Euclidean section where $\delta>0$ and hence $\zeta=\cosh \left(x-x^{\prime}\right)+\frac{1}{2} \mathrm{e}^{x+x^{\prime}} \delta>1$.

Since the modes $\theta_{\lambda}$ form a orthonormal basis in the Hilbert space, Eq. (98) can also be inverted and we obtain the Minkowski Klein-Gordon two-point function on the slice $\Pi_{v}$ by smearing $W_{v}$ against the eigenfunctions $\theta_{\lambda}$. For instance, when $v>1$ this corresponds to the introduction of the fields $\hat{\varphi}_{\lambda}(y)$ on the Minkowskian slice $\Pi_{v}$ obtained by smearing the AdS Klein-Gordon field $\widehat{\Phi}$ with the complete set of modes (100):

$$
\hat{\varphi}_{\lambda}(y)=\int_{-\infty}^{\infty} \widehat{\Phi}(X(x, y)) \theta_{\lambda}(x) \mathrm{e}^{(d-2) x} \mathrm{~d} x .
$$


It can be shown that the field $\hat{\varphi}_{\lambda}(y)$ is a canonical Minkowskian Klein-Gordon field in the Wightman vacuum state. In precise terms, we have that the AdS vacuum expectation value of $\hat{\varphi}_{\lambda}(y)$ is given by

$$
W_{\lambda, \lambda^{\prime}}\left(y, y^{\prime}\right) \equiv\left\langle\Omega\left|\hat{\varphi}_{\lambda}(y) \hat{\varphi}_{\lambda^{\prime}}\left(y^{\prime}\right)\right| \Omega\right\rangle=\delta\left(\lambda-\lambda^{\prime}\right) W_{\lambda}^{M, d}\left(y, y^{\prime}\right) .
$$

In particular, the fields $\hat{\varphi}_{\lambda}$ have zero correlation (and hence commute) for different values of the square mass $\lambda$.

As a specification of the Eqs. (98), when restricting the AdS Klein-Gordon field $\widehat{\Phi}$ to a fixed slice $\Pi_{v}$ (the $d$-dimensional brane) we obtain the following explicit formula for the Källen-Lehmann decomposition of the field in the Minkowskian slice

$$
W_{v}^{d+1}\left(X(x, y), X^{\prime}\left(x, y^{\prime}\right)\right)=\int_{0}^{\infty} \frac{\mathrm{d} \lambda}{2} \mathrm{e}^{-d x}\left[J_{v}\left(\sqrt{\lambda} \mathrm{e}^{-x}\right)\right]^{2} W_{\lambda}^{M, d}\left(y, y^{\prime}\right) .
$$

This formula is telling us that a free field $\Phi$ propagating in the ambient gravitational background will be seen on the $d$-dimensional brane as a superposition of fields with a continuous spectrum of masses but different relative weight given by

$$
\mathrm{d} \mu(\lambda, x)=\frac{\mathrm{d} \lambda}{2} \mathrm{e}^{-d x}\left[J_{v}\left(\sqrt{\lambda} \mathrm{e}^{-x}\right)\right]^{2} .
$$

The results of this section can be used to construct other two-point functions $W_{v}^{d+1,(\varkappa)}(X(x$, $\left.y), X\left(x^{\prime}, y^{\prime}\right)\right)$ for a Klein-Gordon field on AdS by using the other self-adjoint extensions: however, it is not guaranteed that such $W_{v}^{d+1,(\varkappa)}$ can be extended to the other half of AdS since the definition uses the set of coordinates defined only on one half. Moreover, one should prove (or disprove) the AdS invariance and analyticity properties of such states. We will not go any further in this direction in this paper.

\section{Conclusions}

We have considered a particular foliation of a Lorentzian manifold by means of Lorentzian submanifolds over a Riemannian base: such foliation also gives a particular orthogonal splitting of the metric tensor. In this context we have considered a quantum field over the total manifold and decomposed it into a bunch of longitudinal quantum fields $\hat{\varphi}_{\lambda}$ and transversal classical modes $\theta_{\lambda}$.

Such decomposition allows us to pick up a specific member of the bunch by a smearing against those transversal classical modes.

This technique has been then successfully applied:

- to the case of Minkowski, foliated by de Sitter $d$-branes or by accelerated world-lines;

- to the case of de Sitter, foliated by lower dimensional de Sitter branes;

- to anti-de Sitter, foliated by Minkowskian branes.

In all these cases the distinguished analyticity properties of the two-point function in the ambient manifold appear to survive this operation of picking out a specific field, giving a QFT on the leaf with those analyticity properties which are advocated independently for the geometry of the brane itself. 
Since the analytic structure of the two-point function is equivalent to the spectral structure of the Hamiltonian of the theory, this procedure can be regarded as a method for enforcing certain spectral properties on a manifold by embedding it into another manifold where the spectral properties are easier to formulate (this is the case de Sitter $\hookrightarrow$ Minkowski).

Or else we can construct a QFT with certain spectral properties in the ambient manifold by means of the spectral properties of the QFT in the brane (this is the case Minkowski $\hookrightarrow$ AdS).

We also point out that, in more geometrical terms, to some extent what we have done in the examples is decomposing a certain irreducible unitary representation of the invariance group of the ambient manifold into irreducible unitary representations of a certain subgroup which is the invariance group of a submanifold. This might turn out to be of utility in application to representation theory and special functions: indeed some of the relations (e.g., Eqs. (56), (75)) that we have found, relating the two-point functions of the ambient manifold and those of the submanifold are integral representations which are not to be found in the more mathematically oriented literature.

This decomposition has been made here only for the warped-product manifolds for practical computational issues but nevertheless the idea of inheriting spectral properties from an ambient manifold could be extended to other cases, most importantly the Schwarzschild geometry $[5,6]$.

Potentially this perspective is the more appealing the harder is the problem of consistently formulating a spectral property in curved backgrounds.

Additionally, the recent topic raised in $[12,13]$ allows a direct application of this method to general warped $d$-branes in various gravitational backgrounds.

It is our intention to pursue this direction in further publications.

\section{References}

[1] G.W. Gibbons, S.W. Hawking, Phys. Rev. D 15 (1977) 2738.

[2] J. Bros, U. Moschella, Two-point functions and de Sitter quantum fields, Rev. Math. Phys. 8 (1996) 327; gr-qc/9511019.

[3] J. Bros, U. Moschella, J.P. Gazeau, Quantum field theory in the de Sitter universe, Phys. Rev. Lett. 73 (1994) 1746.

[4] J. Bros, H. Epstein, U. Moschella, Analyticity properties and thermal effects for general quantum field theory on de Sitter space-time, Commun. Math. Phys. 196 (1998) 535.

[5] M. Bertola, Effetti termici della quantizzazione in uno spazio-tempo curvo, Laurea Thesis, University of Milan, 1995.

[6] S. Deser, O. Levin, Mapping Hawking into Unruh thermal properties, Phys. Rev. D 59 (1999).

[7] R.F. Streater, A.S. Wightman, PCT, Spin and Statistics, and All That, Benjamin, New York, 1964.

[8] C. Itzykson, J.B. Zuber, Quantum Field Theory, McGraw-Hill, New York, 1988.

[9] N.D. Birrell, P.C.W. Davies, Quantum Fields in Curved Space, Cambridge University Press, Cambridge, 1982.

[10] R.M. Wald, Quantum Field Theory in Curved Spacetime and Black Hole Thermodynamics, The University of Chicago Press, Chicago, 1994. 
[11] M. Bertola, V. Gorini, U. Moschella, R. Schaeffer, Correspondence between Minkowski and de Sitter quantum field theory, Phys. Lett. B 462 (1999) 249-253; hep-th/9906035.

[12] L. Randall, R. Sundrum, A large mass hierarchy from a small extra dimension, Phys. Rev. Lett. 83 (1999) 3370-3373; hep-th/9905221.

[13] S.B. Giddings, E. Katz, L. Randall, Linearized gravity in brane background, hep-th/0002091.

[14] M. Bertola, D. Gouthier, Warped products with special Riemannian curvature, SISSA 118/97/GEO.

[15] M. Bertola, D. Gouthier, Lie triple systems and warped products, SISSA 68/97/GEO, to appear in Rendiconti Università la Sapienza, Rome.

[16] M. Bertola, D. Gouthier, Rigidification of pseudo-Riemannian manifolds by an elliptic equation, SISSA 72/99/GEO.

[17] A.O.G. Källen, Properties of vacuum expectation values of field operators, in: Ecole d'Eté de Physique Théorique, Les Houches, France, 1960.

[18] J. Maldacena, The large $N$ limit of superconformal field theories and supergravity, Adv. Theor. Math. Phys. 2 (1998) 231; hep-th/9711200.

[19] M. Bertola, J. Bros, U. Moschella, R. Schaeffer, AdS/CFT correspondence for $n$-point functions, hep-th/9908140.

[20] M. Reed, B. Simon, Fourier analysis and self-adjointness, in: Methods of Modern Mathematical Physics, Vol. 2, Academic Press, 1975.

[21] B. O’Neill, Semi-Riemannian geometry, in: Pure and Applied Mathematics, Academic Press, 1983.

[22] P. Tondeur, Foliations on Riemannian Manifolds, Springer-Verlag.

[23] J. Bros, G.A. Viano, Connection between the harmonic analysis on the sphere and the harmonic analysis on the one-sheeted hyperboloid: an analytic continuation viewpoint, Forum Math. 8 (1996) 621-658; Forum Math. 8 (1996) 659-722; Forum Math. 9 (1997) 165-191.

[24] H. Bateman, Higher Transcendental Functions, McGraw-Hill, 1954.

[25] T.S. Bunch, P.C.W. Davies, Quantum field theory in de Sitter space: renormalization by point splitting, Proc. R. Soc. London A 360 (1978) 117.

[26] I.S. Gradshteyn, I.M. Ryzhik, Table of Integrals, Series and Products, Academic Press, New York, 1980.

[27] U. Moschella, J. Schaeffer, Quantum fluctuations in the open universe, Phys. Rev. D (1997).

[28] E.C. Titchmarsh, Eigenfunction Expansion Associated with Second Order Differential Equations, Oxford at the Clarendon Press, 1962.

[29] H. Bateman, Tables of Integral Transforms, McGraw-Hill, 1954.

[30] J. Bros, H. Epstein, U. Moschella, in preparation. 